\title{
Homogenization of stiff plates and two-dimensional high-viscosity Stokes equations
}

\author{
Marc BRIANE \\ Institut de Recherche Mathématique de Rennes \\ Université Européenne de Bretagne \\ mbriane@insa-rennes.fr
}

\author{
Juan CASADO-DÍAZ \\ Dpto. de Ecuaciones Diferenciales y Análisis Numérico \\ Universidad de Sevilla \\ jcasadod@us.es
}

April 4, 2012

\begin{abstract}
The paper deals with the homogenization of rigid heterogeneous plates. Assuming that the coefficients are equi-bounded in $L^{1}$, we prove that the limit of a sequence of plate equations remains a plate equation which involves a strongly local linear operator acting on the second gradients. This compactness result is based on a div-curl lemma for fourthorder equations. On the other hand, using an intermediate stream function we deduce from the plates case a similar result for high-viscosity Stokes equations in dimension two, so that the nature of the Stokes equation is preserved in the homogenization process. Finally, we show that the $L^{1}$-boundedness assumption cannot be relaxed. Indeed, in the case of the Stokes equation the concentration of one very rigid strip on a line induces the appearance of second gradient terms in the limit problem, which violates the compactness result obtained under the $L^{1}$-boundedness condition.
\end{abstract}

Keywords : Homogenization, plate, Stokes equation, div-curl lemma

Mathematics Subject Classification : 35B27 - 74Q15 - 76M50

\section{Introduction}

Since the seminal work of Khruslov [17] extended in $[1,11,13]$, it is known that the homogenization of sequences of scalar conductivity equations with high-contrast coefficients may induce nonlocal effects in dimension three. In fact, Mosco [21] showed that the limits - in the sense of $\Gamma$-convergence endowed with the $L^{2}$ strong topology - of the associated diffusion energies are Dirichlet forms which can be represented thanks to the Beurling-Deny [2] formula involving naturally a nonlocal term. On the other hand, Camar-Eddine and Seppecher [13] obtained that the closure of the set of diffusion energies agrees with the set of regular Dirichlet forms. However, dimension two prevents from the appearance of nonlocal effects, since limits of equi-coercive diffusion energies are shown to be purely diffusive $[5,7]$.

The case of vector-valued problems is much more intricate. As a matter of fact, the lack of truncation principle implies that the Beurling-Deny representation formula does not apply. Such important is the absence of constraints that Camar-Eddine and Seppecher [14] proved that in dimension three any objective (vanishing on rigid displacements) lower semi-continuous quadratic functional can be derived as a limit of isotropic elastic energies. As in the scalar 
case dimension two seems at first sight to be more favorable. Indeed, a sequence of twodimensional equi-coercive elastic quadratic functionals is shown [10] to converge to a similar elastic functional provided that the sequence of rigidity tensors is bounded in $L^{1}$. However, contrary to the scalar case this compactness result cannot be improved significantly, since the counter-example of [10] shows that very rigid strips may induce second gradient terms in the limit energy as in dimension three.

It is then natural to study other systems in dimension two. From the Mechanics point of view the asymptotic analysis of plates is relevant. The homogenization of heterogeneous plates with a periodic structure was performed rigorously in [16]. This work was extended in [15] to the non-periodic case including a 2D-3D dimension reduction approach, and using a variant of the $H$-convergence method [22]. In these papers the elastic coefficients are assumed to be equibounded from below and above. Up to our knowledge the homogenization of plates with non uniformly bounded coefficients has not be studied. It seems to be also interesting to compare the plate case to the above mentioned two-dimensional elasticity case under the high-contrast assumption. This is the aim of the present work.

Let $\Omega$ be a bounded open set of $\mathbb{R}^{2}$, and let $A_{n}$ be a sequence of equi-coercive (in the weak sense of (2.3), see also Remark 2.2) symmetric fourth-order tensors in $L^{\infty}\left(\Omega ; \mathcal{L}_{s}\left(\mathbb{R}_{s}^{2 \times 2}\right)\right)$. In section 2 we study the asymptotic behavior of the sequence of the plate equations

$$
\operatorname{div}^{2}\left(A_{n} D^{2} u_{n}\right)=f_{n} \text { in } \Omega,
$$

where $f_{n}$ strongly converges to $f$ in $H^{-2}(\Omega)$, simultaneously with the asymptotic behavior of the associated energies

$$
F_{n}^{\Omega}(u)=\int_{\Omega} A_{n} D^{2} u: D^{2} u d x, \quad \text { for } u \in H^{2}(\Omega)
$$

Assuming that the sequence $\left|A_{n}\right|$ is bounded in $L^{1}(\Omega)$ and converges weakly-* to a Radon measure $\mu$, we prove (see Theorems 2.5, 2.6, and Remark 2.7) that the limit equation of (1.3) is still a plate equation of the type

$$
\operatorname{div}^{2}\left(\mathcal{A}_{\Omega} D^{2} u \mu\right)=f \text { in } \Omega
$$

where $\mathcal{A}_{\Omega}$ is a strongly local linear operator mapping the set of the admissible second gradients into $L_{\mu}^{1}\left(\Omega ; \mathcal{L}_{s}\left(\mathbb{R}_{s}^{2 \times 2}\right)\right)$. This limit behavior is actually independent of the boundary conditions imposed to (1.1). Moreover, when $u$ is regular, we get that $\mathcal{A}_{\Omega} D^{2} u=A D^{2} u \mu$-a.e. in $\Omega$, where the symmetric fourth-order tensor $A$ satisfies: $|A| \leq 1 \mu$-a.e. in $\Omega$, and $A(\mathrm{n} \odot \mathrm{n}):(\mathrm{n} \odot \mathrm{n})=0$ $\mu$-a.e. along any smooth curve with outer normal $\mathrm{n}$ (see Proposition 2.8). The tensor-valued measure $A \mu$ is also proved to vanish on sets of zero capacity. These properties imply that not any equi-coercive symmetric fourth-order tensor can be attained. In terms of energy the sequence $F_{n}^{\Omega}$, with domain $H_{0}^{2}(\Omega)$, is shown to $\Gamma$-converge for the strong topology of $L^{2}(\Omega)$ to a quadratic functional $F^{\Omega}$ which satisfies (see also Remark 2.9)

$$
F^{\Omega}(u)=\int_{\Omega} A D^{2} u: D^{2} u d \mu, \quad \text { for any } u \in C_{c}^{1}(\Omega), \text { piecewise } C^{2} \text { in } \Omega .
$$

The proof of this compactness result uses two ingredients: a suitable div-curl lemma and the $\Gamma$-convergence approach. On the one hand, the div-curl lemma (see Theorem 2.11) allows us to pass to the limit in the product $A_{n} D^{2} u_{n}: D^{2} v_{n}$, when $u_{n}$ solves the plate equation (1.1) and $F_{n}^{\Omega}\left(v_{n}\right)$ is bounded. This result is an extension to fourth-order equations of the div-curl 
lemmas for second-order equations established in [6] (scalar case) and in [10] (elasticity case). It is based on the embedding of the set of matrix-valued measures which are div ${ }^{2}$ free, into $H^{-1}\left(\Omega ; \mathbb{R}^{2 \times 2}\right.$ ), involving the Strauss inequality [23] (see Lemma 2.13), and the concentration compactness lemma of P.-L. Lions [20]. On the other hand, similarly to [6] the $\Gamma$-convergence approach provides a functional framework given by the domain of the $\Gamma$-limit of the energies (1.2). However, two difficulties arise in this approach. Firstly, contrary to the diffusion case studied in [7] there is no truncation principle. Secondly, the fourth-order character of the equations makes delicate the localization with a regular cut-off function $\varphi$. Indeed, to this end we should consider sequences of the type $A_{n} D^{2}\left(v_{n} \varphi\right): D^{2}\left(v_{n} \varphi\right)$, with $F_{n}^{\Omega}\left(v_{n}\right)$ bounded, for which we cannot estimate the term $A_{n}\left(\nabla u_{n} \odot \nabla \varphi\right):\left(\nabla u_{n} \odot \nabla \varphi\right)$, since $\left|A_{n}\right|$ is only bounded in $L^{1}(\Omega)$. So, the proof of Theorems 2.5 is split in nine steps using several fourth-order equations with Dirichlet or Neumann boundary conditions and various quadratic functionals, and needs several back and forth in order to overcome these difficulties.

Taking into account the compactness result for the plates case, in Section 3 we study the homogenization of the Stokes equations

$$
\left\{\begin{array}{l}
-\operatorname{Div}\left(B_{n} D u_{n}\right)+\nabla p_{n}=g_{n} \text { in } \Omega \\
\operatorname{div} u_{n}=0 \text { in } \Omega,
\end{array}\right.
$$

where the sequence $g_{n}$ strongly converges to $g$ in $H^{-1}(\Omega)$, and $B_{n}$ is a sequence of equi-coercive (in the sense of (3.3)) matrix-valued viscosities in $L^{\infty}\left(\Omega ; \mathcal{L}_{s}\left(\mathbb{R}_{0}^{2 \times 2}\right)\right)$. Assuming that $\left|B_{n}\right|$ is bounded in $L^{1}(\Omega)$ and converges weakly-* to a Radon measure $\nu$, we prove (see Theorems 3.5, 3.6, and Remark 3.7) that the homogenized equation of (1.5) is given by

$$
\left\{\begin{array}{l}
-\operatorname{Div}\left(\mathcal{B}_{\Omega} D u \nu\right)+\nabla p=g \text { in } \Omega \\
\operatorname{div} u=0 \text { in } \Omega,
\end{array}\right.
$$

where $\mathcal{B}_{\Omega}$ is a local linear operator mapping the set of admissible gradients into $L_{\mu}^{1}\left(\Omega ; \mathcal{L}_{s}\left(\mathbb{R}_{0}^{2 \times 2}\right)\right)$. Moreover, when $u$ is regular, we obtain that $\mathcal{B}_{\Omega} D u=B D u \nu$-a.e. in $\Omega$, where the symmetric fourth-order tensor $B$ satisfies: $|B| \leq 1 \nu$-a.e. in $\Omega$, and $B(\mathrm{t} \odot \mathrm{n}):(\mathrm{t} \odot \mathrm{n})=0 \nu$-a.e. along any smooth curve with tangent $\mathrm{t}$ and outer normal $\mathrm{n}$.

Defining $A_{n}$ by $A_{n} \xi=-J B_{n}(J \xi)$ for $\xi \in \mathbb{R}_{s}^{2 \times 2}$, where $J$ is the rotation matrix of $90^{\circ}$ angle, the previous compactness result is easily deduced from the plates case, while the convergence of the pressure $p_{n}$ in $H_{\mathrm{loc}}^{-1}(\Omega) / \mathbb{R}$ is a consequence of [8].

Finally in Section 4, we show in the case of the Stokes equation that the $L^{1}$-boundedness of the coefficients cannot be relaxed. Following [10] we build a counter-example with rigid strips, but here we consider one strip concentrated on a line. We obtain (see Theorem 4.1) a homogenized problem with second gradient terms, which thus does not belong to the class of equations (1.6). Due to the incompressibility condition, our approach differs radically from the one used in the elasticity case of [10]. In particular, the proof involves surprisingly a wave equation (see Lemma 4.2).

\section{$1.1 \quad$ Notations}

- $I$ denotes the unit matrix of $\mathbb{R}^{2 \times 2}$, and $J=\left(\begin{array}{cc}0 & -1 \\ 1 & 0\end{array}\right)$.

- : denotes the scalar product in $\mathbb{R}^{2 \times 2}$, i.e. $\xi: \eta=\operatorname{tr}\left(\xi^{T} \eta\right)$ for any $\xi, \eta \in \mathbb{R}^{2 \times 2}$. 
- $\mathbb{R}_{s}^{2 \times 2}$ denotes the set of the $(2 \times 2)$ symmetric real matrices, and $\mathcal{L}_{s}\left(\mathbb{R}_{s}^{2 \times 2}\right)$ the set of the fourth-order tensors $A$ mapping $\mathbb{R}_{s}^{2 \times 2}$ into itself, which are symmetric, i.e. $A \xi: \eta=A \eta: \xi$ for any $\xi, \eta \in \mathbb{R}_{s}^{2 \times 2}$.

- $\mathbb{R}_{0}^{2 \times 2}$ denotes the set of $(2 \times 2)$ real matrices with zero trace, and $\mathcal{L}_{s}\left(\mathbb{R}_{0}^{2 \times 2}\right)$ the set of the symmetric fourth-order tensors mapping $\mathbb{R}_{0}^{2 \times 2}$ into itself.

- $\odot$ denotes the symmetric tensorial product, i.e., for any $x, y \in \mathbb{R}^{2}, x \odot y$ is the matrix of $\mathbb{R}_{s}^{2 \times 2}$ with entries $(x \odot y)_{i j}=\frac{1}{2}\left(x_{i} y_{j}+x_{j} y_{i}\right), 1 \leq i, j \leq 2$.

- $\nabla u$ denotes the gradient of the scalar distribution $u$, and $D^{2} u$ is the symmetric Hessian matrix of the second-order derivatives of $u$.

- $D U$ denotes the Jacobian matrix of the vector-valued distribution $U=\left(U_{1}, U_{2}\right)$, the rows of which are $\nabla U_{1}, \nabla U_{2}$, and $e(U)=\frac{1}{2}\left(D U+D U^{T}\right)$ is the symmetrized gradient of $U$.

- div denotes the classical divergence operator acting on the vector-valued distributions, Div denotes the vector-valued differential operator taking the divergence of each row of matrix-valued distributions, and $\operatorname{div}^{2}$ denotes the operator div (Div).

- For a bounded open set $\omega$ of $\mathbb{R}^{2}, H_{\text {div }}^{1}(\omega)$ and $H_{0, \text { div }}^{1}(\omega)$ denote the space of the divergence free vector-valued functions in $H^{1}\left(\omega ; \mathbb{R}^{2}\right)$ and $H_{0}^{1}\left(\omega ; \mathbb{R}^{2}\right)$ respectively.

- $\mathcal{M}(\Omega)$ denotes the space of bounded Radon measures on $\Omega$, and $\stackrel{*}{\longrightarrow}$ denotes the weak-* convergence in $\mathcal{M}(\Omega)$, considered as the dual of the space $C_{0}^{0}(\Omega)$ of continuous functions vanishing on $\partial \Omega$.

- A curve of class $C^{2}$ in $\mathbb{R}^{2}$ is the range of a mapping $\eta \in C^{2}\left(0,1 ; \mathbb{R}^{2}\right)$ such that $\eta^{\prime}(t) \neq 0$ for any $t \in(0,1)$. Sometimes, we will also consider $C^{2}$ curves as mapping in $C^{2}\left([0,1] ; \mathbb{R}^{2}\right)$.

- For a bounded set $O$ of $\mathbb{R}^{2}$ and a bounded open set $\tilde{\Omega}$ such that $\bar{O} \subset \tilde{\Omega}$, the capacity of $O$ relating to $\tilde{\Omega}$ is defined by

$$
\operatorname{Cap}(O, \tilde{\Omega})=\inf \left\{\int_{\tilde{\Omega}}|\nabla \varphi|^{2} d x: \varphi \in H_{0}^{1}(\tilde{\Omega}), \varphi \geq 1 \text { a.e. in a neighborhood of } O\right\} .
$$

Clearly, with this definition, the capacity of $O$ depends on the set $\Omega$, but the zero capacity does not depend on $\tilde{\Omega}$. Recall that the elements of the Sobolev space $H^{1}(\Omega)$ have a continuous representative defined up to a set of zero capacity. Moreover, any Radon measure in $H^{-1}(\Omega)$ vanishes on sets of zero capacity.

\subsection{Recalls of $\Gamma$-convergence}

In this section we recall the definition of the De Giorgi $\Gamma$-convergence and some of its properties which will be used in the sequel. We refer to [12] for an exhaustive presentation of $\Gamma$-convergence (see also [4] for an elementary approach).

Definition 1.1. Let $d, N$ be two positive integers, and let $\omega$ be a bounded open set of $\mathbb{R}^{d}$. A sequence of functionals $F_{n}: L^{2}(\omega)^{N} \rightarrow[0, \infty]$ is said to $\Gamma$-converge to $F: L^{2}(\omega)^{N} \rightarrow[0, \infty]$ for the strong topology of $L^{2}(\omega)^{N}$, if for any $u$ in $L^{2}(\omega)^{N}$,

i) the $\Gamma$-liminf inequality holds

$$
\forall u_{n} \rightarrow u \quad \text { in } L^{2}(\omega)^{N}, \quad F(u) \leq \liminf _{n \rightarrow \infty} F_{n}\left(u_{n}\right),
$$


ii) the $\Gamma$-limsup inequality holds

$$
\exists \bar{u}_{n} \rightarrow u \text { in } L^{2}(\omega)^{N}, \quad F(u)=\lim _{n \rightarrow \infty} F_{n}\left(\bar{u}_{n}\right) .
$$

Any sequence satisfying (1.8) will be called a recovery sequence (for $F_{n}$ ) of limit $u$.

Properties 1.2. The following properties hold:

a) Since $L^{2}(\omega)^{N}$ is separable, any sequence of functionals $F_{n}: L^{2}(\omega)^{N} \rightarrow[0, \infty]$ has a subsequence which $\Gamma$-converges with respect to the strong topology of $L^{2}(\omega)^{N}$.

b) Let $F_{n}: L^{2}(\omega)^{N} \rightarrow[0, \infty]$ be a sequence of quadratic forms which $\Gamma$-converges to $F$. Then, $F$ is a quadratic form on $L^{2}(\omega)^{N}$ which is lower semi-continuous with respect to the topology of $L^{2}(\omega)^{N}$. Moreover, the domain $D(F)$ of $F$ is a Hilbert space endowed with the norm

$$
\|u\|_{D(F)}=\left(\|u\|_{L^{2}(\omega)^{N}}^{2}+F(u)\right)^{\frac{1}{2}} .
$$

c) Let $F_{n}: L^{2}(\omega)^{N} \rightarrow[0, \infty]$ be a sequence of quadratic forms which $\Gamma$-converges to $F$. Let $\Phi_{n}$ be the bilinear form associated with $F_{n}$ on its domain. Then, for any $u \in L^{2}(\omega)^{N}$, with $F(u)<\infty$, a sequence $u_{n}$ converging to $u$ in $L^{2}(\omega)^{N}$, with $F_{n}\left(u_{n}\right)$ bounded, is a recovery sequence (1.8) (for $F_{n}$ ), if and only if

$$
\forall v_{n} \rightarrow 0 \quad \text { in } L^{2}(\omega)^{N}, \text { with } F_{n}\left(v_{n}\right) \leq c, \quad \lim _{n \rightarrow \infty} \Phi_{n}\left(u_{n}, v_{n}\right)=0 .
$$

In the sequel, we will always consider the $\Gamma$-convergence for the strong topology of $L^{2}(\omega)^{N}$. This topology will be not necessarily mentioned. Indeed, all the functionals $F_{n}$ will have the property that any sequence $u_{n}$ bounded in $L^{2}(\omega)^{N}$, with $F_{n}\left(u_{n}\right)$ bounded, is compact in $L_{\text {loc }}^{2}(\omega)^{N}$. In this framework the weak convergence in $L^{2}(\omega)^{N}$ agrees with the weak convergence in $L^{2}(\omega)^{N}$ combined with the strong convergence in $L_{\mathrm{loc}}^{2}(\omega)^{N}$.

\section{Homogenization of plates}

\subsection{Statement of the results}

Consider a bounded connected open set $\Omega$ of $\mathbb{R}^{2}$ and a sequence $A_{n} \in L^{\infty}\left(\Omega ; \mathcal{L}_{s}\left(\mathbb{R}_{s}^{2 \times 2}\right)\right)$ such that

$$
\begin{gathered}
\left|A_{n}\right| \stackrel{*}{\longrightarrow} \mu \quad \text { in } \mathcal{M}(\Omega), \\
A_{n}(x) \xi: \xi \geq 0, \quad \forall \xi \in \mathbb{R}_{s}^{2 \times 2}, \text { a.e. } x \in \Omega
\end{gathered}
$$

and such that for any open sets $O, \omega$ with $\bar{O} \subset \omega \subset \Omega$, there exists a constant $\alpha(O, \omega)$ satisfying

$$
\alpha(O, \omega) \int_{O}\left|D^{2} u\right|^{2} d x \leq \int_{\omega}|u|^{2} d x+\int_{\omega} A_{n} D^{2} u: D^{2} u d x, \quad \forall u \in H^{2}(\omega) .
$$

The purpose of the present section is to study the asymptotic behavior of a sequence of functions $u_{n} \in H^{2}(\omega)$, for an open set $\omega \subset \Omega$, which solve the plate equation

$$
\operatorname{div}^{2}\left(A_{n} D^{2} u_{n}\right)=f_{n} \text { in } \omega,
$$

for some compact sequence $f_{n} \in H^{-2}(\omega)$, and which satisfy the limits

$$
u_{n} \rightarrow u \text { in } L^{2}(\omega), \quad \limsup _{n \rightarrow \infty} \int_{\omega} A_{n} D^{2} u_{n}: D^{2} u_{n} d x<\infty,
$$

for some $u \in L^{2}(\Omega) \cap H_{\text {loc }}^{2}(\Omega)$. 
Remark 2.1. Observe that no boundary condition is imposed in (2.4). We will show actually that the limit equation for (2.4) does not depend on the boundary conditions.

Remark 2.2. We have preferred assuming that $A_{n}$ satisfies the local integral ellipticity condition (2.3) since several classical elliptic operators for the plate equations (as for example the heterogeneous bi-Laplacian operator $\left.\Delta\left(a_{n} \Delta\right)\right)$ do not usually satisfy a strong pointwise ellipticity condition of the type

$$
A_{n}(x) \xi: \xi \geq \alpha|\xi|^{2}, \quad \forall \xi \in \mathbb{R}_{s}^{2 \times 2} \text {, a.e. } x \in \omega, \forall n \in \mathbb{N},
$$

for some constant $\alpha>0$. Thanks to the local $H^{2}$-estimates for the second-order elliptic equations (see, e.g., [18] Theorem 9.11), in order to have (2.3) it is enough to assume that there exists a relatively compact sequence of matrix functions $M_{n}$ in $C^{0}\left(\Omega ; \mathbb{R}_{s}^{2 \times 2}\right)$, with $M_{n} \geq \alpha I$ for some positive constant $\alpha$, such that $A_{n}$ satisfies

$$
A_{n}(x) \xi: \xi \geq\left|M_{n}(x): \xi\right|^{2}, \quad \forall \xi \in \mathbb{R}_{s}^{2 \times 2}, \text { a.e. } x \in \omega, \forall n \in \mathbb{N} \text {. }
$$

So, the bi-Laplacian $\Delta\left(a_{n} \Delta\right)$ satisfies $(2.7)$ with $M_{n}=a_{n} I$, when $a_{n} \geq \alpha$ a.e. in $\omega$.

Remark 2.3. Thanks to estimate (2.3) it is possible to solve some partial differential problems associated with the diffusion operators $\operatorname{div}^{2}\left(A_{n} D^{2} u\right)$. In this sense observe that for any open set $\omega \subset \Omega$ and any $h \in L^{2}(\omega)$, there exists a unique solution $u$ of the Neumann problem

$$
\left\{\begin{array}{l}
\operatorname{div}^{2}\left(A_{n} D^{2} u\right)+u=h \text { in } \omega \\
\left(A_{n} D^{2} u\right) \mathrm{n}=0, \operatorname{Div}\left(A_{n} D^{2} u\right) \cdot \mathrm{n}=0 \text { on } \partial \omega
\end{array}\right.
$$

or equivalently, of the minimization problem

$$
\min \left\{\frac{1}{2} \int_{\omega}\left(A_{n} D^{2} v: D^{2} v+|v|^{2}\right) d x-\int_{\omega} h v: v \in H_{\mathrm{loc}}^{2}(\omega) \cap L^{2}(\omega)\right\} .
$$

On the other hand, for an open set $\omega$ strictly contained in $\Omega$, extending by zero outside $\Omega$ the functions of $H_{0}^{2}(\omega)$ and applying inequality (2.3), with $\omega$ replaced by $\Omega$ and $O$ replaced by $\omega$, we deduce that $A_{n}$ satisfies

$$
\alpha(\omega, \Omega) \int_{\omega}\left|D^{2} u\right|^{2} d x \leq \int_{\omega} A_{n} D^{2} u: D^{2} u d x+\int_{\omega}|u|^{2}, \quad \forall u \in H_{0}^{2}(\omega),
$$

Therefore, for any $f \in H^{-2}(\omega)$, we can solve the Dirichlet problem

$$
\left\{\begin{array}{l}
\operatorname{div}^{2}\left(A_{n} D^{2} u\right)+u=f \text { in } \omega \\
u \in H_{0}^{2}(\omega)
\end{array}\right.
$$

We will use the following:

Definition 2.4. For any open set $\omega \subset \Omega$, denote by $\mathcal{H}(\omega)$ the space of the functions $u \in H^{2}(\omega)$ such that there exists a sequence $u_{n} \in H^{2}(\omega)$ satisfying $(2.5)$, and by $\mathcal{H}_{c}(\omega)$ the space of the functions $u \in \mathcal{H}(\omega)$ such that there exists a sequence $u_{n} \in H^{2}(\omega)$ satisfying $(2.5)$, with $\operatorname{supp}\left(u_{n}\right)$ contained in a compact subset of $\omega$ independent of $n$. Also denote by $D^{2} \mathcal{H}(\omega)$ and $D^{2} \mathcal{H}_{c}(\omega)$ the spaces defined by

$$
D^{2} \mathcal{H}(\omega)=\left\{D^{2} u: u \in \mathcal{H}(\omega)\right\} \quad \text { and } \quad D^{2} \mathcal{H}_{c}(\omega)=\left\{D^{2} u: u \in \mathcal{H}_{c}(\omega)\right\}
$$


Our first result on the asymptotic behavior of the solutions of (2.4) is the following:

Theorem 2.5. There exists a subsequence of $n$, still denoted by $n$, and a family of symmetric bilinear mappings $\mathfrak{a}_{\omega}: D^{2} \mathcal{H}(\omega) \times D^{2} \mathcal{H}(\omega) \rightarrow \mathcal{M}(\omega)$, for any open set $\omega \subset \Omega$, with the following properties:

- The operator $\mathfrak{a}_{\omega}$ is strongly local with respect to $\omega$ in the following sense: If $\omega^{1}, \omega^{2}$ are two open sets of $\omega$ and $u^{1}, v^{1} \in \mathcal{H}\left(\omega^{1}\right), u^{2}, v^{2} \in \mathcal{H}\left(\omega^{2}\right)$ are such that $D^{2} u^{1}=D^{2} u^{2}$, $D^{2} v^{1}=D^{2} v^{2}$ a.e. in $\omega^{1} \cap \omega^{2}$, then we have

$$
\mathfrak{a}_{\omega^{1}}\left(D^{2} u^{1}, D^{2} v^{1}\right)=\mathfrak{a}_{\omega^{2}}\left(D^{2} u^{2}, D^{2} v^{2}\right) \text { in } \omega^{1} \cap \omega^{2}
$$

- For any open set $\omega \subset \Omega$, and $u_{n}, u \in H^{2}(\omega)$ satisfying (2.4) and (2.5) for some sequence $f_{n}$ converging strongly to $f$ in $H^{-2}(\omega)$, the function $u$ is solution of

$$
\int_{\omega} d \mathfrak{a}_{\omega}\left(D^{2} u, D^{2} v\right)=\langle f, u\rangle, \quad \forall v \in \mathcal{H}_{c}(\omega) .
$$

- Consider an open set $\omega \subset \Omega$, and $u_{n}, u \in H^{2}(\omega)$ satisfying (2.5) and the convergences

$$
\int_{\omega} A_{n} D^{2} u_{n}: D^{2} v_{n} d x \longrightarrow 0
$$

for any $v_{n} \in H^{2}(\omega)$, with support in a fixed compact of $\omega$, such that

$$
v_{n} \rightarrow 0 \text { in } L^{2}(\omega), \quad \limsup _{n \rightarrow \infty} \int_{\omega} A_{n} D^{2} v_{n}: D^{2} v_{n} d x<\infty .
$$

Then, we have

$$
A_{n} D^{2} u_{n}: D^{2} v_{n} \stackrel{*}{\longrightarrow} \mathfrak{a}_{\omega}\left(D^{2} u, D^{2} v\right) \text { in } \mathcal{M}(\omega),
$$

for any $v_{n}, v$ in $H^{2}(\omega)$ such that

$$
v_{n} \rightarrow v \quad \text { in } L^{2}(\omega), \quad \limsup _{n \rightarrow \infty} \int_{\omega} A_{n} D^{2} v_{n}: D^{2} v_{n} d x<\infty .
$$

- For any open set $\omega \subset \Omega$, the sequence of functionals $G_{n}^{\omega}$ defined by

$$
G_{n}^{\omega}(u)= \begin{cases}\int_{\omega} A_{n} D^{2} u: D^{2} u d x & \text { if } u \in H_{0}^{2}(\omega) \\ \infty & \text { if } u \in L^{2}(\omega) \backslash H_{0}^{2}(\omega) .\end{cases}
$$

$\Gamma$-converges (up to a subsequence) to a functional $G^{\omega}$ which satisfies

$$
G^{\omega}(u)=\int_{\omega} d \mathfrak{a}_{\omega}\left(D^{2} u, D^{2} u\right), \quad \forall u \in \mathcal{H}_{c}(\omega) .
$$

- The family $\mathfrak{a}_{\omega}$ satisfies the following ellipticity condition: For any open sets $O, U, \omega$ with $\bar{O} \subset U \subset \bar{U} \subset \omega \subset \Omega$, we have

$$
\alpha(O, U) \int_{O}\left|D^{2} u\right|^{2} d x \leq \int_{\omega}|u|^{2} d x+\int_{\omega} d \mathfrak{a}_{\omega}\left(D^{2} u: D^{2} u\right), \quad \forall u \in H^{2}(\omega) .
$$


- For any open set $\omega \subset \Omega$ and any $u \in W^{2, \infty}(\omega)$, we have

$$
\int_{\omega} d \mathfrak{a}_{\omega}\left(D^{2} u, D^{2} u\right) \leq \mu(\omega)\left\|D^{2} u\right\|_{L^{\infty}\left(\omega ; \mathbb{R}_{s}^{2 \times 2}\right)}^{2} .
$$

- The family $a_{\omega}$ satisfies the following continuity property: For any open set $\omega \subset \Omega$ and any sequence $u^{k} \in \mathcal{H}(\omega)$ which converges to some $u \in \mathcal{H}(\omega)$ in $L^{2}(\omega)$, with $\mathfrak{a}_{\omega}\left(D^{2} u^{k}, D^{2} u^{k}\right)$ bounded in $\mathcal{M}(\omega)$, we have

$$
\mathfrak{a}_{\omega}\left(D^{2} u^{k}, D^{2} v\right) \stackrel{*}{\longrightarrow} \mathfrak{a}_{\omega}\left(D^{2} u, D^{2} v\right) \quad \text { in } \mathcal{M}(\omega), \quad \forall v \in \mathcal{H}(\omega) .
$$

For the case of smooth functions, it is possible to obtain an integral representation of $\mathfrak{a}_{\omega}$. This is given by the following result:

Theorem 2.6. For any open set $\omega \subset \Omega$, there exists a linear operator $\mathcal{A}_{\omega}: D^{2} \mathcal{H}(\omega) \rightarrow$ $L_{\mu}^{1}\left(\omega ; \mathbb{R}_{s}^{2 \times 2}\right)$, with the following properties:

- The operator $\mathcal{A}_{\omega}$ is strongly local with respect to $\omega$ in the following sense: If $\omega^{1}, \omega^{2}$ are two open subsets of $\Omega$ and $u^{1} \in \mathcal{H}\left(\omega^{1}\right), u^{2} \in \mathcal{H}\left(\omega^{2}\right)$ are such that $D^{2} u^{1}=D^{2} u^{2}$ a.e. in $\omega^{1} \cap \omega^{2}$, then

$$
\mathcal{A}_{\omega^{1}} D^{2} u^{1}=\mathcal{A}_{\omega^{2}} D^{2} u^{2} \quad \mu \text {-a.e. in } \omega^{1} \cap \omega^{2} .
$$

- The operators $\mathfrak{a}_{\omega}$ and $\mathcal{A}_{\omega}$ are related by

$$
\mathfrak{a}_{\omega}\left(D^{2} u, D^{2} v\right)=\mathcal{A}_{\omega} D^{2} u: D^{2} v \mu, \quad \forall u, v \in \mathcal{H}(\omega), v \in C^{2}(\omega) .
$$

- $\mathcal{A}_{\omega} D^{2} u \mu$ vanishes on sets of zero capacity and satisfies

$$
\int_{\omega}\left|\mathcal{A}_{\omega} D^{2} u\right| \varphi d \mu \leq\left(\int_{\omega} \varphi d \mathfrak{a}_{\omega}\left(D^{2} u, D^{2} u\right)\right)^{\frac{1}{2}}\left(\int_{\omega} \varphi d \mu\right)^{\frac{1}{2}}, \forall \varphi \in C_{0}^{0}(\omega), \varphi \geq 0 .
$$

Moreover, there exists a non-negative tensor $A \in L_{\mu}^{\infty}\left(\Omega ; \mathcal{L}_{s}\left(\mathbb{R}_{s}^{2 \times 2}\right)\right)$, with

$$
\|A\|_{L_{\mu}^{\infty}\left(\Omega ; \mathcal{L}_{s}\left(\mathbb{R}_{s}^{2 \times 2}\right)\right)} \leq 1
$$

such that for any open set $\omega \subset \Omega$ and any $u \in C^{2}(\omega) \cap \mathcal{H}^{2}(\omega)$, we have

$$
\mathcal{A}_{\omega} D^{2} u=A D^{2} u \quad \mu \text {-a.e. in } \omega \text {. }
$$

Remark 2.7. A straightforward consequence of Theorems 2.5 and 2.6 is that for any open set $\omega \subset \Omega$, and for any $u_{n}, u \in H^{2}(\omega)$ satisfying (2.4), (2.5) for some sequence $f_{n}$ converging strongly to $f$ in $H^{-2}(\omega)$, the limit $u$ solves the equation

$$
\operatorname{div}^{2}\left(\mathcal{A}_{\omega} D^{2} u \mu\right)=f \text { in } \omega .
$$

In the case where $u$ belongs to $C^{2}(\bar{\omega})$, this equation can be written as

$$
\operatorname{div}^{2}\left(A D^{2} u \mu\right)=f \text { in } \omega .
$$

Equations (2.24) and (2.21) give a representation of the operators $\mathfrak{a}_{\omega}$ for functions of class $C^{2}$. This representation formula holds true actually for functions which are less smooth. A result in this sense is the following one: 
Proposition 2.8. For any open curve $\gamma \subset \Omega$ of class $C^{2}$, the tensor $A \in L_{\mu}^{\infty}\left(\omega ; \mathcal{L}_{s}\left(\mathbb{R}_{s}^{2 \times 2}\right)\right)$ given by Theorem 2.6 satisfies

$$
A(\mathrm{n} \odot \mathrm{n}):(\mathrm{n} \odot \mathrm{n})=0 \quad \text { p-a.e. in } \gamma,
$$

where $\mathrm{n}$ is the unitary normal to $\gamma$.

Let $\omega$ be an open set of $\Omega$, and let $O_{1}, O_{2}$ be two disjoint open sets of class $C^{2}$ such that

$$
\bar{\omega}=\overline{\omega \cap O_{1}} \cup \overline{\omega \cap O_{2}} \text {. }
$$

Then, for any $u \in C^{1}(\omega)$, with $u \in C^{2}\left(\omega \cap \overline{O_{1}}\right) \cap C^{2}\left(\omega \cap \overline{O_{2}}\right)$, equality (2.24) holds true.

Remark 2.9. For any function $u \in C^{1}(\omega)$, with $u \in C^{2}\left(\omega \cap \overline{O_{1}}\right) \cap C^{2}\left(\omega \cap \overline{O_{2}}\right)$, the derivatives

$$
\frac{\partial^{2} u}{\partial \mathrm{t}^{2}} \text { and } \frac{\partial^{2} u}{\partial \mathrm{t} \partial \mathrm{n}}
$$

where $\mathrm{t}$ is a tangent vector to $\partial O_{1} \cap \partial O_{2}$, are uniquely defined on $\partial O_{1} \cap \partial O_{2}$. Thus, thanks to (2.25) $A D^{2} u$ is well defined for the measure $\mu$. Moreover, such a function $u$ with a compact support in $\omega$ belongs clearly to $\mathcal{H}_{c}(\omega)$ due to the boundedness of $\left|A_{n}\right|$ in $L^{1}(\omega)$. Therefore, the representation formula of the $\Gamma$-limit (2.16) holds true for any $u \in C_{c}^{1}(\omega)$, which is piecewise $C^{2}$ in $\omega$.

Remark 2.10. Since for any $u \in \mathcal{H}^{2}(\omega), \mathcal{A}_{\omega} D^{2} u \mu$ vanishes on sets of zero capacity, the measure $\mathcal{A}_{\omega} D^{2} u \mu$ does not charge single points.

The proof of our results will be based on the following div-curl type result:

Theorem 2.11. Consider an open set $\omega \subset \mathbb{R}^{2}$, and sequences $\Lambda_{n} \in L^{2}\left(\omega ; \mathbb{R}_{s}^{2 \times 2}\right), v_{n} \in H^{2}(\omega)$. Assume the existence of measures $\lambda, \nu \in \mathcal{M}(\omega), \Lambda \in \mathcal{M}\left(\omega ; \mathbb{R}_{s}^{2 \times 2}\right)$, and of a function $v \in H^{2}(\omega)$, satisfying the convergences

$$
\begin{gathered}
\Lambda_{n} \stackrel{*}{\longrightarrow} \Lambda \text { in } \mathcal{M}\left(\omega ; \mathbb{R}_{s}^{2 \times 2}\right), \quad \operatorname{div}^{2} \Lambda_{n} \rightarrow \operatorname{div}^{2} \Lambda \text { in } H^{-2}(\omega), \quad\left|\Lambda_{n}-\Lambda\right| \stackrel{*}{\longrightarrow} \lambda \text { in } \mathcal{M}(\omega), \\
v_{n} \rightarrow v \text { in } H^{2}(\omega), \quad\left|D^{2}\left(v_{n}-v\right)\right| \stackrel{*}{\longrightarrow} \nu \text { in } \mathcal{M}(\omega) .
\end{gathered}
$$

Then, there exist a constant $C>0$ independent of $n$, and sequences $\left(x_{i}\right)_{i \in \mathbb{N}}$ in $\omega,\left(a_{i}\right)_{i \in \mathbb{N}}$ in $\mathbb{R}^{2}$, satisfying

$$
\left|a_{i}\right| \leq C \lambda\left(\left\{x_{i}\right\}\right) \nu\left(\left\{x_{i}\right\}\right)^{\frac{1}{2}}, \quad \forall i \in \mathbb{N},
$$

such that for any $\varphi \in C_{c}^{\infty}(\omega)$, the following limit holds

$$
\begin{aligned}
& \lim _{n \rightarrow \infty} \int_{\omega} \Lambda_{n}: D^{2} v_{n} \varphi d x=\left\langle\operatorname{div}^{2} \Lambda, v \varphi\right\rangle_{H^{-2}(\omega), H_{0}^{2}(\omega)} \\
& -2\langle\Lambda, \nabla v \odot \nabla \varphi\rangle_{H^{-1}\left(\omega ; \mathbb{R}_{s}^{2 \times 2}\right), H_{0}^{1}\left(\omega ; \mathbb{R}_{s}^{2 \times 2}\right)}-\int_{\omega} v D^{2} \varphi: d \Lambda+\sum_{i=0}^{\infty} a_{i} \cdot \nabla \varphi\left(x_{i}\right) .
\end{aligned}
$$

When $v$ belongs to $C^{2}(\omega)$, (2.30) can be written as

$$
\Lambda_{n}: D^{2} v_{n} \stackrel{*}{\longrightarrow} \Lambda: D^{2} v-\operatorname{div}\left(\sum_{i=0}^{\infty} a_{i} \delta_{x_{i}}\right) \quad \text { in } \mathcal{D}^{\prime}(\omega) .
$$


Corollary 2.12. If in lemma 2.11 the sequence $\Lambda_{n}: D^{2} v_{n}$ is bounded in $L^{1}(\omega)$ and the function $v$ belongs to $C^{2}(\omega)$, then we have

$$
\Lambda_{n}: D^{2} v_{n} \stackrel{*}{\longrightarrow} \Lambda: D^{2} v \quad \text { in } \mathcal{M}(\omega)
$$

Theorem 2.11 is a consequence of the following two lemmas. The first one is based on results of [8] (which are themselves based on [3] and [23]). In particular it gives a sense to the second term of the right-hand side of (2.30). The second one is based on the previous one and the P.L. Lions concentration compactness [20].

Lemma 2.13. For any smooth bounded open set $\omega \subset \mathbb{R}^{2}$, the space $\mathcal{M}_{\operatorname{div}^{2}}\left(\omega ; \mathbb{R}_{s}^{2 \times 2}\right)$ of matrixvalued functions $\Lambda \in \mathcal{M}\left(\omega ; \mathbb{R}_{s}^{2 \times 2}\right)$ satisfying

$$
\operatorname{div}^{2}(\Lambda)=0 \text { in } \omega
$$

is continuously embedded in $H^{-1}\left(\omega ; \mathbb{R}_{s}^{2 \times 2}\right)$.

Lemma 2.14. Consider a smooth bounded open set $\omega \subset \mathbb{R}^{2}$, and measures $\Lambda_{n} \in \mathcal{M}_{\operatorname{div}^{2}}\left(\omega ; \mathbb{R}_{s}^{2 \times 2}\right)$, $\lambda \in \mathcal{M}(\omega)$ satisfying the convergences

$$
\Lambda_{n} \stackrel{*}{\longrightarrow} 0 \text { in } \mathcal{M}\left(\omega ; \mathbb{R}_{s}^{2 \times 2}\right), \quad\left|\Lambda_{n}\right| \stackrel{*}{\longrightarrow} \lambda \text { in } \mathcal{M}(\omega)
$$

Define $Z_{n}$ as the solution of

$$
\left\{\begin{array}{l}
-\Delta Z_{n}=\Lambda_{n} \text { in } \omega \\
Z_{n} \in H_{0}^{1}\left(\omega ; \mathbb{R}_{s}^{2 \times 2}\right) .
\end{array}\right.
$$

Then, there exist a constant $C>0$, and sequences $\left(x_{i}\right)_{i \in \mathbb{N}}$ in $\omega,\left(a_{i}\right)_{i \in \mathbb{N}}$ in $\mathbb{R}^{2}$, satisfying

$$
\left|a_{i}\right| \leq C \lambda\left(\left\{x_{i}\right\}\right)^{2}, \quad \forall i \in \mathbb{N}
$$

such that, up to extract a subsequence, the following limit holds

$$
\left|\nabla Z_{n}\right|^{2} \stackrel{*}{\longrightarrow} \sum_{i=0}^{\infty} a_{i} \delta_{x_{i}} \text { in } \mathcal{M}(\omega) .
$$

\subsection{Proof of the results}

Proof of Lemma 2.13. It is easy to check that for any $\Lambda \in \mathcal{M}_{\operatorname{div}^{2}}\left(\omega ; \mathbb{R}_{s}^{2 \times 2}\right)$, with entries $\Lambda_{i j}$, $i, j \in\{1,2\}$, the matrix-valued measure $Q \Lambda$ defined as

$$
Q \Lambda=\left(\begin{array}{cc}
-\Lambda_{12} & -\Lambda_{22} \\
\Lambda_{11} & \Lambda_{12}
\end{array}\right)
$$

has zero trace and satisfies $\operatorname{curl}(\operatorname{Div}(Q \Lambda))=0$ in $\omega$. The result then follows from [8] in which it is proved that the space of matrix-valued measures $\Upsilon$ with zero trace and satisfying $\operatorname{curl}(\operatorname{Div}(\Upsilon))=0$ in $\omega$, is continuously imbedded in $H^{-1}\left(\omega ; \mathbb{R}_{s}^{2 \times 2}\right)$. In the present twodimensional case this can be deduced from the Strauss theorem [23]. In higher dimension it is based on the work of Bourgain, Brezis [3].

Proof of Lemma 2.14. First we remark that since $\Lambda_{n}$ tends to zero in $\mathcal{M}_{\operatorname{div}^{2}}(\omega)$, Lemma 2.13 implies that $Z_{n}$ converges weakly to zero in $H_{0}^{1}\left(\omega ; \mathbb{R}_{s}^{2 \times 2}\right)$. 
Fix $\varphi \in C_{c}^{1}(\omega)$, putting $Z_{n} \varphi^{2}$ as test function in (2.35), we have

$$
\begin{aligned}
& \sum_{i, j=1}^{2}\left(\int_{\omega}\left|\nabla\left(Z_{n}\right)_{i j}\right|^{2} \varphi^{2} d x+2 \int_{\omega}\left(Z_{n}\right)_{i j} \varphi \nabla\left(Z_{n}\right)_{i j} \cdot \nabla \varphi d x\right) \\
& =\left\langle\Lambda_{n} \varphi, Z_{n} \varphi\right\rangle_{H^{-1}\left(\omega ; \mathbb{R}_{s}^{2 \times 2}\right), H_{0}^{1}\left(\omega ; \mathbb{R}_{s}^{2 \times 2}\right)} \leq\left\|\Lambda_{n} \varphi\right\|_{H^{-1}\left(\omega ; \mathbb{R}_{s}^{2 \times 2}\right)}\left\|Z_{n} \varphi\right\|_{H_{0}^{1}\left(\omega ; \mathbb{R}_{s}^{2 \times 2}\right)}
\end{aligned}
$$

In this inequality the weak convergence to zero of $\nabla\left(Z_{n}\right)_{i j}$ in $L^{2}\left(\omega ; \mathbb{R}^{N}\right)$ and the RellichKondrachov compactness theorem imply that

$$
\begin{gathered}
\sum_{i, j=1}^{2} \int_{\omega}\left(Z_{n}\right)_{i j} \varphi \nabla\left(Z_{n}\right)_{i j} \cdot \nabla \varphi d x \longrightarrow 0 \\
\left\|Z_{n} \varphi\right\|_{H_{0}^{1}\left(\omega ; \mathbb{R}_{s}^{2 \times 2}\right)}-\left(\sum_{i, j=1}^{2} \int_{\omega}\left|\nabla\left(Z_{n}\right)_{i j}\right|^{2} \varphi^{2} d x\right)^{\frac{1}{2}} \longrightarrow 0 .
\end{gathered}
$$

Now, fix $q \in(1,2)$. Since $\Lambda_{n}$ converges weakly-* to zero in the measures sense, $\Lambda_{n}$ also converges strongly to zero in $W^{-1, q}(\omega)$. Hence, the sequence

$$
\zeta_{n}=\operatorname{div}^{2}\left(\Lambda_{n} \varphi\right)=\sum_{i, j=1}^{2}\left(2 \partial_{i}\left(\Lambda_{n}\right)_{i j} \partial_{j} \varphi+\left(\Lambda_{n}\right)_{i j} \partial_{i j}^{2} \varphi\right)
$$

converges strongly to zero in $W^{-2, q}(\omega)$. Therefore, there exists $H_{n} \in L^{q}\left(\omega ; \mathbb{R}_{s}^{2 \times 2}\right)$ converging strongly to 0 in $L^{q}\left(\omega ; \mathbb{R}_{s}^{2 \times 2}\right)$ such that $\operatorname{div}^{2}\left(H_{n}\right)=\zeta_{n}$. Using that

$$
\operatorname{div}^{2}\left(\Lambda_{n} \varphi-H_{n}\right)=0 \text { in } \omega
$$

we can apply Lemma 2.13 to deduce

$$
\left\|\Lambda_{n} \varphi-H_{n}\right\|_{H^{-1}\left(\omega ; \mathbb{R}_{s}^{2 \times 2}\right)} \leq C\left\|\Lambda_{n} \varphi-H_{n}\right\|_{\mathcal{M}\left(\omega ; \mathbb{R}_{s}^{2 \times 2}\right)} .
$$

Taking into account that the strong convergence of $H_{n}$ to 0 in $L^{q}\left(\omega ; \mathbb{R}_{s}^{2 \times 2}\right)$ implies strong convergence in $\mathcal{M}\left(\omega ; \mathbb{R}_{s}^{2 \times 2}\right)$ and in $H^{-1}\left(\omega ; \mathbb{R}_{s}^{2 \times 2}\right)$, the previous estimate yields

$$
\limsup _{n \rightarrow \infty}\left\|\Lambda_{n} \varphi\right\|_{H^{-1}\left(\omega ; \mathbb{R}_{s}^{2 \times 2}\right)} \leq C \lim _{n \rightarrow \infty}\left\|\Lambda_{n} \varphi\right\|_{\mathcal{M}\left(\omega ; \mathbb{R}_{s}^{2 \times 2}\right)}=C \int_{\omega}|\varphi| d \lambda
$$

Using (2.39), (2.40) and (2.41) in (2.38), and denoting by $z$ the limit of $\left|\nabla Z_{n}\right|^{2}$ in the weak-* sense of the measures (which exists at least for a subsequence), we have just proved that

$$
\int_{\omega}|\varphi|^{2} d z \leq C\left(\int_{\omega}|\varphi| d \lambda\right)^{2}, \quad \forall \varphi \in C_{c}^{1}(\omega) .
$$

Finally, thanks to Lemma 1.3 in [20], there exist two sequences $\left(x_{i}\right)_{i \in \mathbb{N}}$ in $\omega$ and $\left(a_{i}\right)_{i \in \mathbb{N}}$ in $\mathbb{R}^{2}$, satisfying (2.36), such that

$$
z=\sum_{i=0}^{\infty} a_{i} \delta_{x_{i}}
$$

which implies the desired limit (2.37). 
Proof of Theorem 2.11. For any $\varphi \in C_{c}^{\infty}(\omega)$, we have

$$
\begin{aligned}
& \left\langle\operatorname{div}^{2} \Lambda_{n}, v_{n} \varphi\right\rangle_{H^{-2}(\omega), H_{0}^{2}(\Omega)} \\
& =\int_{\omega} \varphi \Lambda_{n}: D^{2} v_{n} d x+2 \int_{\omega} \Lambda_{n}:\left(\nabla v_{n} \odot \nabla \varphi\right) d x+\int_{\omega} v_{n} \Lambda_{n}: D^{2} \varphi d x .
\end{aligned}
$$

In this equality the strong convergence of $\operatorname{div}^{2} \Lambda_{n}$ to $\operatorname{div}^{2} \Lambda$ in $H^{-2}(\omega)$, the weak-* convergence of $\Lambda_{n}$ to $\Lambda$ in $\mathcal{M}\left(\omega ; \mathbb{R}_{s}^{2 \times 2}\right)$, and the weak convergence of $v_{n}$ to $v$ in $H^{2}(\omega)$, together with the compact embedding of $H^{2}(\omega)$ into $C_{0}^{0}(\bar{\omega})$ imply that

$$
\begin{aligned}
\int_{\omega} v_{n} \Lambda_{n}: D^{2} \varphi d x & \longrightarrow \int_{\omega} v D^{2} \varphi: d \Lambda \\
\left\langle\operatorname{div}^{2} \Lambda_{n}, v_{n} \varphi\right\rangle_{H^{-2}(\omega), H_{0}^{2}(\omega)} & \longrightarrow\left\langle\operatorname{div}^{2} \Lambda, v \varphi\right\rangle_{H^{-2}(\omega), H_{0}^{2}(\omega)}
\end{aligned}
$$

In order to pass to the limit in the second term of the right-hand side of (2.42), we remark that since $\operatorname{div}^{2}\left(\Lambda_{n}-\Lambda\right)$ converges strongly to zero in $H^{-2}(\omega)$, there exists a sequence $H_{n}$ converging strongly to zero in $L^{2}\left(\omega ; \mathbb{R}_{s}^{2 \times 2}\right)$ such that $\operatorname{div}^{2}\left(\Lambda_{n}-\Lambda-H_{n}\right)=0$ in $\omega$. Consider the solution $Z_{n}$ of

$$
\left\{\begin{array}{l}
-\Delta Z_{n}=\Lambda_{n}-\Lambda-H_{n} \text { in } \omega \\
Z_{n} \in H_{0}^{1}\left(\omega ; \mathbb{R}_{s}^{2 \times 2}\right)
\end{array}\right.
$$

Then, we have

$$
\begin{aligned}
& 2 \int_{\omega} \Lambda_{n}:\left(\nabla v_{n} \odot \nabla \varphi\right) d x \\
& =\sum_{i, j=1}^{2} \int_{\omega}\left(\nabla\left(Z_{n}\right)_{i j} \cdot\left(\nabla \partial_{i} v_{n} \partial_{j} \varphi+\nabla \partial_{j} v_{n} \partial_{i} \varphi\right)+\nabla\left(Z_{n}\right)_{i j} \cdot\left(\partial_{i} v_{n} \nabla \partial_{j} \varphi+\partial_{j} v_{n} \nabla \partial_{i} \varphi\right)\right) d x \\
& +2\left\langle\Lambda+H_{n}, \nabla v_{n} \odot \nabla \varphi\right\rangle_{H^{-1}\left(\omega ; \mathbb{R}_{s}^{2}\right), H_{0}^{1}\left(\mathbb{R}_{s}^{2}\right)},
\end{aligned}
$$

Since $Z_{n}$ converges weakly to zero in $H_{0}^{1}\left(\omega ; \mathbb{R}_{s}^{2 \times 2}\right)$ by Lemma $2.13, \nabla v_{n}$ converges weakly to $\nabla v$ in $H^{1}\left(\omega ; \mathbb{R}^{2}\right)$ and thus strongly in $L^{2}\left(\omega ; \mathbb{R}_{s}^{2 \times 2}\right)$, and since $H_{n}$ converges strongly to zero in $L^{2}\left(\omega ; \mathbb{R}_{s}^{2 \times 2}\right)$, we have

$$
\begin{gathered}
\sum_{i, j=1}^{2} \int_{\omega} \nabla\left(Z_{n}\right)_{i j} \cdot\left(\partial_{i} v_{n} \nabla \partial_{j} \varphi+\partial_{j} v_{n} \nabla \partial_{j} \varphi\right) d x \longrightarrow 0 \\
2\left\langle\Lambda+H_{n}, \nabla v_{n} \odot \nabla \varphi\right\rangle_{H^{-1}\left(\omega ; \mathbb{R}_{s}^{2}\right), H_{0}^{1}\left(\mathbb{R}_{s}^{2}\right)} \longrightarrow 2\langle\Lambda, \nabla v \odot \nabla \varphi\rangle_{H^{-1}\left(\omega ; \mathbb{R}_{s}^{2}\right), H_{0}^{1}\left(\mathbb{R}_{s}^{2}\right)} .
\end{gathered}
$$

On the other hand, from Lemma 2.14 above combined with Lemma 2.11 of [9], we easily deduce that

$$
\sum_{i, j=1}^{2} \int_{\omega} \nabla\left(Z_{n}\right)_{i j} \cdot\left(\nabla \partial_{i} v_{n} \partial_{j} \varphi+\nabla \partial_{j} v_{n} \partial_{j} \varphi\right) d x \longrightarrow-\sum_{i=0}^{\infty} a_{i} \cdot \nabla \varphi\left(x_{i}\right)
$$

for some sequences $\left(x_{i}\right)_{i \in \mathbb{N}}$ in $\omega$ and $\left(a_{i}\right)_{i \in \mathbb{N}}$ in $\mathbb{R}^{2}$, which do not depend on $\varphi$ and satisfy (2.29). Therefore, we have

$$
2 \int_{\omega} \Lambda_{n}:\left(\nabla v_{n} \odot \nabla \varphi\right) d x \longrightarrow 2\langle\Lambda, \nabla v \odot \nabla \varphi\rangle_{H^{-1}\left(\omega ; \mathbb{R}_{s}^{2}\right), H_{0}^{1}\left(\mathbb{R}_{s}^{2}\right)}-\sum_{i=0}^{\infty} a_{i} \cdot \nabla \varphi\left(x_{i}\right)
$$


Finally, substituting (2.43), (2.44) and (2.46) in (2.42) we get (2.30).

Proof of Corollary 2.12. The boundness of $\Lambda_{n}: D^{2} v_{n}$ in $L^{1}(\omega)$ implies that the convergence (2.31) holds actually in the weak-* sense of the measures on $\omega$. As $\Lambda: D^{2} v$ is a measure on $\omega$, so is the distribution

$$
\operatorname{div}\left(\sum_{i=0}^{\infty} a_{i} \delta_{x_{i}}\right)
$$

This implies that $a_{i}=0$, for any $i \in \mathbb{N}$. Hence, (2.31) gives (2.32).

Proof of Theorem 2.5. To obtain the representation formula for the limit of the operator $\operatorname{div}^{2}\left(A_{n} D^{2}\right)$, we need to make several back and forth, since we cannot use directly trial functions as $\varphi u_{n}$, for $\varphi \in C_{c}^{\infty}(\Omega)$, in the $\Gamma$-convergence process (see the Introduction). In particular, we are led to first study the case of the open sets which are strictly contained in $\Omega$. The proof is divided in nine steps:

- In Step 1 we prove that for any open ball $B \subset \Omega$, there exists a subsequence still denoted by $n$, such that for any recovery sequence $u_{n}$ of limit $u$ associated with the sequence of energies with density $A_{n} D^{2} u_{n}: D^{2} u_{n}$ in $B$, we get that $A_{n} D^{2} u_{n}: D^{2} u_{n}$ converges weakly-* in $\mathcal{M}(B)$ to some Radon measure $\nu_{u, u}^{B}$ which is quadratic in $u$.

- In Step 2 we prove that the measure $\nu_{u, u}^{B}$ only depends on the second gradient $D^{2} u$.

- Step 3 allows us to define in any open set $\omega \subset \Omega$, the limit energy $\mathfrak{a}_{\omega}\left(D^{2} u, D^{2} u\right)$, and to prove simultaneously the strong locality property $(2.9)$ satisfied by the operator $\mathfrak{a}_{\omega}$.

- Step 4 is devoted to the proof of the continuity of the linear mapping $u \in \mathcal{H}(\omega) \mapsto$ $\mathfrak{a}_{\omega}\left(D^{2} u, D^{2} v\right)$, for any $v$ in the set $\mathcal{H}(\omega)$ of the admissible trial functions (see Definition 2.4).

- In Step 5 we obtain the limit variational formulation (2.10) associated with the sequence of equations $\operatorname{div}^{2}\left(A_{n} D^{2}\right)=f_{n}$, for any strongly convergent sequence $f_{n}$ in $H^{-2}(\omega)$.

- In Step 6 we derive the representation formula (2.16) for the $\Gamma$-limit of the sequence $G_{n}^{\omega}$ defined by $(2.15)$, in any open set $\omega$ strictly contained in $\Omega$.

- In Step 7 we establish for any open set $O \subset \bar{O} \subset \Omega$, the convergence of $A_{n} D^{2} u_{n}: D^{2} v_{n}$ to $\mathfrak{a}_{\omega}\left(D^{2} u, D^{2} v\right)$ weakly-* in $\mathcal{M}(\omega)$, for suitable bounded energy sequences $u_{n}, v_{n}$ converging respectively to $u, v$. In Step 8 this convergence is extended to the case of an arbitrary open set $\omega \subset \Omega$.

- Finally, Step 9 is devoted to the proofs of the bound from below (2.17), and the bound from above (2.18) satisfied by the limit density energy $\mathfrak{a}_{\omega}\left(D^{2} u, D^{2} u\right)$, for any open set $\omega \subset \Omega$.

Step 1. Consider an open ball $B \subset \Omega$, and for any $n \in \mathbb{N}$, the functional $F_{n}^{B}: L^{2}(B) \rightarrow \mathbb{R}$ defined by

$$
F_{n}^{B}(u)= \begin{cases}\int_{B} A_{n} D^{2} u: D^{2} u d x & \text { if } u \in H^{2}(B) \\ \infty & \text { if } u \in L^{2}(B) \backslash H^{2}(B) .\end{cases}
$$


By Properties 1.2 of $\Gamma$-convergence, we know that, for a subsequence of $n$, the $\Gamma$-limit $F^{B}$ of $F_{n}^{B}$ does exist. It is a quadratic functional the domain of which $D\left(F^{B}\right)$ is a Hilbert space endowed with the norm given by (1.9).

Since by $(2.1), C^{2}(\bar{B})$ is contained in $D\left(F^{B}\right)$, the domain $D\left(F^{B}\right)$ is continuously and densely embedded in $L^{2}(B)$. Then, by a duality argument $L^{2}(B)$ is continuously and densely embedded in the dual $D\left(F^{B}\right)^{\prime}$. Thus, denoting by $\Phi^{B}$ the bilinear form associated with $F^{B}$ and taking a countable dense subset $\left\{h^{k}\right\}$ of $L^{2}(B)$, the functions $w^{k} \in D\left(F^{B}\right)$ defined through the Riesz theorem by

$$
\left\{\begin{array}{l}
w^{k} \in D\left(F^{B}\right) \\
\Phi^{B}\left(w^{k}, v\right)+\int_{B} w^{k} v d x=\int_{B} h^{k} v d x, \quad \forall v \in D\left(F^{B}\right),
\end{array}\right.
$$

form a dense subset of $D\left(F^{B}\right)$. Moreover, the solution $w_{n}^{k} \in H^{2}(B)$ of the Neumann problem

$$
\left\{\begin{array}{l}
\operatorname{div}^{2}\left(A_{n} D^{2} w_{n}^{k}\right)+w_{n}^{k}=h^{k} \text { in } B, \\
\left(A_{n} D^{2} w_{n}^{k}\right) \mathrm{n}=0, \operatorname{Div}\left(A_{n} D^{2} w_{n}^{k}\right) \cdot \mathrm{n}=0 \text { on } \partial B,
\end{array}\right.
$$

with $\mathrm{n}$ the unitary outer normal to $B$ on $\partial B$, is a recovery sequence (for $F_{n}^{B}$ ) of limit $w^{k}$ for any $k \in \mathbb{N}$. By a diagonal argument, we can extract another subsequence of $n$, such that the following limit holds

$$
A_{n} D^{2} w_{n}^{k}: D^{2} w_{n}^{k} \stackrel{*}{\longrightarrow} \nu^{k} \text { in } \mathcal{M}(B), \quad \forall k \in \mathbb{N} .
$$

Now, consider $u \in D\left(F^{B}\right)$ and a recovery sequence $u_{n}$ (for $F_{n}^{B}$ ) of limit $u$. Extracting another subsequence if necessary, we can assume that there exists a non-negative measure $\nu^{B} \in \mathcal{M}(B)$, such that $A_{n} D^{2} u_{n}: D^{2} u_{n}$ converges weakly-* to $\nu^{B}$ in $\mathcal{M}(B)$. On the other hand, taking a sequence $w^{k_{j}}$ converging to $u$ in $D\left(F^{B}\right)$, and using that $u_{n}+w_{n}^{k_{j}}, u_{n}-w_{n}^{k_{j}}$ are recovery sequences (for $F_{n}^{B}$ ) of limits $u+w^{k_{j}}, u-w^{k_{j}}$ respectively, we have

$$
\begin{aligned}
& \left\|\nu^{B}-\nu^{k_{j}}\right\|_{\mathcal{M}(B)} \leq \liminf _{n \rightarrow \infty} \int_{B}\left|A_{n} D^{2} u_{n}: D^{2} u_{n}-A_{n} D^{2} w_{n}^{k_{j}}: D^{2} w_{n}^{k_{j}}\right| d x \\
& \leq \lim _{n \rightarrow \infty}\left(\int_{B} A_{n} D^{2}\left(u_{n}+w_{n}^{k_{j}}\right): D^{2}\left(u_{n}+w_{n}^{k_{j}}\right) d x\right)^{\frac{1}{2}}\left(\int_{B} A_{n} D^{2}\left(u_{n}-w_{n}^{k_{j}}\right): D^{2}\left(u_{n}-w_{n}^{k_{j}}\right) d x\right)^{\frac{1}{2}} \\
& =\left(F^{B}\left(u+w^{k_{j}}\right)\right)^{\frac{1}{2}}\left(F^{B}\left(u-w^{k_{j}}\right)\right)^{\frac{1}{2}} .
\end{aligned}
$$

Therefore, $\nu^{k_{j}}$ converges strongly to $\nu^{B}$ in $\mathcal{M}(B)$. As a consequence, the whole sequence $A_{n} D^{2} u_{n}: D^{2} u_{n}$ converges to $\nu^{B}$, which only depends on $u$. Since, the map $u \in D\left(F^{B}\right) \mapsto \nu^{B} \in$ $\mathcal{M}(B)$ is quadratic, we may denote the measure $\nu^{B}$ associated with $u \in D\left(F^{B}\right)$ as $\nu_{u, u}^{B}$. We also have

$$
\left\|\nu_{u, u}^{B}\right\|_{\mathcal{M}(B)} \leq F^{B}(u) .
$$

Hence, the mapping $u \in D\left(F^{B}\right) \mapsto \nu_{u, u}^{B} \in \mathcal{M}(B)$ is continuous.

Step 2. Let $\left(B_{m}\right)_{m \in \mathbb{N}}$ be a countable family of open balls strictly contained in $\omega$, such that any open subset of $\Omega$ contains a ball of the family. Using a diagonal procedure we can apply Step 1 to any ball of the family, with a subsequence of $n$ independent of $m$.

Now, consider an open set $\omega \subset \Omega$, and $u_{n}, u \in H^{2}(\omega)$, satisfying (2.4) and (2.5) for some compact sequence $f_{n} \in H^{-2}(B)$. Let $\hat{u} \in H^{2}\left(B_{m}\right)$ be a function such that $D^{2} u=D^{2} \hat{u}$ in $B_{m}$. Since $u_{n}+\hat{u}-u$ converges strongly to $\hat{u}$ in $L^{2}\left(B_{m}\right)$, and

$$
\limsup _{n \rightarrow \infty} \int_{B_{m}} A_{n} D^{2}\left(u_{n}+\hat{u}-u\right): D^{2}\left(u_{n}+\hat{u}-u\right) d x=\limsup _{n \rightarrow \infty} \int_{B_{m}} A_{n} D^{2} u_{n}: D^{2} u_{n} d x<\infty,
$$


the function $\hat{u}$ belongs to $D\left(F^{B_{m}}\right)$. Let $\hat{u}_{n}$ be a recovery sequence (for $F^{B_{m}}$ ) of limit $\hat{u}$. Also consider a sequence $g^{k} \in L^{2}\left(B_{m}\right)$ such that the solution $z^{k} \in D\left(F^{B_{m}}\right)$ of

$$
\Phi^{B_{m}}\left(z^{k}, v\right)+\int_{B_{m}} z^{k} v d x=\int_{B_{m}} g^{k} v d x, \quad \forall v \in D\left(F^{B_{m}}\right),
$$

converges in $D\left(F^{B_{m}}\right)$ to $\hat{u}$. Denote by $z_{n}^{k}$ the solution of the Neumann problem

$$
\left\{\begin{array}{l}
\operatorname{div}^{2}\left(A_{n} D^{2} z_{n}^{k}\right)+z_{n}^{k}=g^{k} \text { in } B_{m} \\
\left(A_{n} D^{2} z_{n}^{k}\right) \mathrm{n}=0, \operatorname{Div}\left(A_{n} D^{2} z_{n}^{k}\right) \cdot \mathrm{n}=0 \text { on } \partial B_{m}
\end{array}\right.
$$

Applying Corollary 2.12 with $\Lambda_{n}=A_{n} D^{2}\left(u_{n}-z_{n}^{k}\right)$ and $v_{n}=u_{n}-\hat{u}_{n}$ (note that the limit $v$ of $v_{n}$ is quadratic thus regular), for any $k \in \mathbb{N}$ and for any $\varphi \in C_{c}^{\infty}\left(B_{m}\right), \varphi \geq 0$, we have

$$
\begin{aligned}
& \limsup _{n \rightarrow \infty} \int_{B_{m}} A_{n} D^{2}\left(u_{n}-\hat{u}_{n}\right): D^{2}\left(u_{n}-\hat{u}_{n}\right) \varphi d x \\
& \leq \lim _{n \rightarrow \infty} \int_{B_{m}} A_{n} D^{2}\left(u_{n}-z_{n}^{k}\right): D^{2}\left(u_{n}-\hat{u}_{n}\right) \varphi d x+\limsup _{n \rightarrow \infty} \int_{B_{m}} A_{n} D^{2}\left(z_{n}^{k}-\hat{u}_{n}\right): D^{2}\left(u_{n}-\hat{u}_{n}\right) \varphi d x \\
& =\limsup _{n \rightarrow \infty} \int_{B_{m}} A_{n} D^{2}\left(z_{n}^{k}-\hat{u}_{n}\right): D^{2}\left(u_{n}-\hat{u}_{n}\right) \varphi d x .
\end{aligned}
$$

Hence, by the Cauchy-Schwartz inequality combining with the fact that $z_{n}^{k}-\hat{u}_{n}$ is a recovery sequence (for $F^{B_{m}}$ ) of limit $z^{k}-\hat{u}$, it follows that

$$
\begin{aligned}
& \limsup _{n \rightarrow \infty} \int_{B_{m}} A_{n} D^{2}\left(u_{n}-\hat{u}_{n}\right): D^{2}\left(u_{n}-\hat{u}_{n}\right) \varphi d x \\
& \leq\|\varphi\|_{L^{\infty}\left(B_{m}\right)}^{\frac{1}{2}}\left(F^{B_{m}}\left(z^{k}-\hat{u}\right)\right)^{\frac{1}{2}} \limsup _{n \rightarrow \infty}\left(\int_{B_{m}} A_{n} D^{2}\left(u_{n}-\hat{u}_{n}\right): D^{2}\left(u_{n}-\hat{u}_{n}\right) \varphi d x\right)^{\frac{1}{2}},
\end{aligned}
$$

which implies that

$$
\limsup _{n \rightarrow \infty} \int_{B_{m}} A_{n} D^{2}\left(u_{n}-\hat{u}_{n}\right): D^{2}\left(u_{n}-\hat{u}_{n}\right) \varphi d x \leq\|\varphi\|_{L^{\infty}\left(B_{m}\right)} F^{B_{m}}\left(z^{k}-\hat{u}\right), \quad \forall k \in \mathbb{N} .
$$

Therefore, passing to the limit as $k$ tends to infinity, we obtain

$$
\lim _{n \rightarrow \infty} \int_{B_{m}} A_{n} D^{2}\left(u_{n}-\hat{u}_{n}\right): D^{2}\left(u_{n}-\hat{u}_{n}\right) \varphi d x=0, \quad \forall \varphi \in C_{c}^{\infty}\left(B_{m}\right), \varphi \geq 0 .
$$

Since $\nu_{\hat{u}, \hat{u}}^{B_{m}}$ is the weak-* limit of $A_{n} D^{2} \hat{u}_{n}: D^{2} \hat{u}_{n}$ in $\mathcal{M}\left(B_{m}\right)$, this shows that

$$
A_{n} D^{2} u_{n}: D^{2} u_{n} \stackrel{*}{\longrightarrow} \nu_{\hat{u}, \hat{u}}^{B_{m}} \text { in } \mathcal{M}\left(B_{m}\right),
$$

for any $\hat{u} \in H^{2}\left(B_{m}\right)$ such that $D^{2} \hat{u}=D^{2} u$ a.e. in $B_{m}$.

Step 3. Consider two balls $B_{l}, B_{m}$ of the family defined in Step 2, with $B_{m} \subset B_{l}$, and the functions $w^{k}, w_{n}^{k}$ defined in Step 1 with $B=B_{l}$. By (2.53), for any $\hat{w}^{k} \in D\left(F^{B_{m}}\right)$ such that $D^{2} w^{k}=D^{2} \hat{w}^{k}$ a.e. in $B_{m}$, we have

$$
\nu_{w^{k}, w^{k}}^{B_{l}}=\lim _{n \rightarrow \infty} A_{n} D^{2} w_{n}^{k}: w_{n}^{k}=\nu_{\hat{w}^{k}, \hat{w}^{k}}^{B_{m}} \text { weakly-* in } \mathcal{M}\left(B_{m}\right) .
$$


Then, by definition of $\nu_{u, u}^{B_{l}}$, and arguing as in Step 1, we obtain that

$$
\nu_{u, u}^{B_{l}}=\nu_{\hat{u}, \hat{u}}^{B_{m}} \text { in } B_{m}, \quad \forall u \in D\left(F^{B_{l}}\right), \forall \hat{u} \in D\left(F^{B_{m}}\right), \text { with } D^{2} \hat{u}=D^{2} u \text { a.e. in } B_{m} .
$$

Taking into account that the family of balls $B_{m}$ is a basis for the topology of $\Omega$, we thus deduce that for any $i, j \in \mathbb{N}$,

$$
\nu_{u, u}^{B_{i}}=\nu_{\hat{u}, \hat{u}}^{B_{j}} \text { in } B_{i} \cap B_{j}, \quad \forall u \in D\left(F^{B_{i}}\right), \forall \hat{u} \in D\left(F^{B_{j}}\right) \text {, with } D^{2} \hat{u}=D^{2} u \text { a.e. in } B_{i} \cap B_{j} .
$$

Therefore, we may define for any $\omega \subset \Omega$, the bilinear form $\mathfrak{a}_{\omega}: D^{2} \mathcal{H}(\omega) \times D^{2} \mathcal{H}(\omega) \rightarrow \mathcal{M}(\omega)$ by

$$
\mathfrak{a}_{\omega}\left(D^{2} u, D^{2} v\right)=\frac{1}{4}\left(\nu_{u+v, u+v}^{B_{m}}-\nu_{u-v, u-v}^{B_{m}}\right) \quad \text { in } B_{m} \cap \omega, \quad \forall m \in \mathbb{N}
$$

so that the strong locality property (2.9) is satisfied.

Step 4. Let $\omega$ be an open set of $\Omega$, and let $u^{k}$ be a sequence in $\mathcal{H}(\omega)$, which converges strongly to a function $u$ in $L^{2}(\omega)$ and such that $\mathfrak{a}_{\omega}\left(D^{2} u^{k}, D^{2} u^{k}\right)$ is bounded in $\mathcal{M}(\omega)$. Then, the sequence $u^{k}$ converges weakly to $u$ in $D\left(F^{B_{m}}\right)$ for any $B_{m}$ contained in $\omega$. Therefore, using that the mapping $u \in D\left(F^{B_{m}}\right) \mapsto \nu_{u, u}^{B_{m}} \in \mathcal{M}\left(B_{m}\right)$ is continuous, the sequence $a_{\omega}\left(D^{2} u^{k}, D^{2} v\right)$ converges weakly-* in $\mathcal{M}\left(B_{m}\right)$ to $a_{\omega}\left(D^{2} u, D^{2} v\right)$, for any $v \in \mathcal{H}^{2}(\omega)$ and for any $m \in \mathbb{N}$. Hence, (2.19) holds.

Step 5. Consider an open set $\omega \subset \Omega$, and $u_{n}, u$ satisfying (2.4) and (2.5) for some $f_{n}$ which converges strongly to $f$ in $H^{-2}(\omega)$. Let us prove that for any $v_{n}$, $v$ satisfying $(2.14)$, we have (2.13). Clearly, it is enough to show that

$$
A_{n} D^{2} u_{n}: D^{2} v_{n} \stackrel{*}{\longrightarrow} \mathfrak{a}_{\omega}\left(D^{2} u, D^{2} v\right) \text { in } \mathcal{M}\left(B_{m}\right), \quad \forall m \in \mathbb{N} \text {, with } B_{m} \subset \omega .
$$

To this end, consider recovery sequences $\hat{u}_{n}$ and $\hat{v}_{n}$ (for $F_{n}^{B_{m}}$ ) of limits $u$ and $v$ respectively. Then, by (2.32) we have

$$
A_{n} D^{2} u_{n}: D^{2}\left(v_{n}-\hat{v}_{n}\right) \stackrel{*}{\longrightarrow} 0 \text { in } \mathcal{M}\left(B_{m}\right)
$$

while by (2.52) we get

$$
A_{n} D^{2}\left(u_{n}-\hat{u}_{n}\right): D^{2} \hat{v}_{n} \stackrel{*}{\longrightarrow} 0 \text { in } \mathcal{M}\left(B_{m}\right)
$$

Therefore, we have

$$
\lim _{n \rightarrow \infty} A_{n} D^{2} u_{n}: D^{2} v_{n}=\lim _{n \rightarrow \infty} A_{n} D^{2} \hat{u}_{n}: D^{2} \hat{v}_{n}=\mathfrak{a}_{\omega}\left(D^{2} u, D^{2} v\right) \text { weakly-* in } \mathcal{M}\left(B_{m}\right),
$$

which gives $(2.55)$.

If now $v$ is in $\mathcal{H}_{c}(\omega)$, then we can take a sequence $v_{n}$ with support in a fixed compact subset $K$ of $\omega$. Then, for any $\varphi \in C_{0}^{0}(\omega)$ such that $\varphi \equiv 1$ in $K$, we have

$$
\int_{\omega} A_{n} D^{2} u_{n}: D^{2} v_{n} d x=\int_{\omega} A_{n} D^{2} u_{n}: D^{2} v_{n} \varphi d x \longrightarrow \int_{\omega} \varphi d \mathfrak{a}\left(D^{2} u, D^{2} v\right) .
$$

Moreover, since $D^{2} v=0$ a.e. in the open set $\omega \backslash K$, the strong locality property (2.9) of $a_{\omega}$ shows that $a_{\omega}\left(D^{2} u, D^{2} v\right)=a_{\omega}\left(D^{2} u, 0\right)=0$ in $\omega \backslash K$. Hence, it follows that

$$
\int_{\omega} A_{n} D^{2} u_{n}: D^{2} v_{n} d x \longrightarrow \int_{\omega} d \mathfrak{a}\left(D^{2} u, D^{2} v\right)
$$


On the other hand, we have

$$
\int_{\omega} A_{n} D^{2} u_{n}: D^{2} v_{n} d x=\left\langle f_{n}, v_{n}\right\rangle \longrightarrow\langle f, v\rangle .
$$

Therefore, we get the limit variational formulation (2.10).

Step 6. For an open set $\omega$ with $\bar{\omega} \subset \Omega$, consider the functional $G_{n}^{\omega}: L^{2}(\omega) \rightarrow \mathbb{R}$ defined by (2.15). We know that, up to a subsequence, $G_{n}^{\omega} \Gamma$-converges to some $G^{\omega}$. Denote by $\Psi^{\omega}$ the bilinear form associated with $G^{\omega}$. As in Step 1, the space $\mathcal{W}^{\omega}$ composed of the functions $w \in D\left(G^{\omega}\right)$ such that there exists $h \in L^{2}(\omega)$ satisfying

$$
\Psi^{\omega}(w, v)+\int_{\omega} w v d x=\int_{\omega} h v d x, \quad \forall v \in D\left(G^{\omega}\right),
$$

is dense in $D\left(G^{\omega}\right)$. Moreover, for any $w \in \mathcal{W}^{\omega}$, the function $w_{n}$ solution of

$$
\left\{\begin{array}{l}
\operatorname{div}^{2}\left(A_{n} D^{2} w_{n}\right)+w_{n}=h \text { in } \omega \\
w_{n} \in H_{0}^{2}(\omega)
\end{array}\right.
$$

is a recovery sequence (for $G_{n}^{\omega}$ ) of limit $w$. On the other hand, by Step 5 the function $w$ satisfies

$$
\int_{\omega} d \mathfrak{a}\left(D^{2} w, D^{2} v\right)+\int_{\omega} w v d x=\int_{\omega} h v d x, \quad \forall v \in \mathcal{H}_{c}(\omega),
$$

which implies that

$$
\Psi^{\omega}(w, v)=\int_{\omega} d \mathfrak{a}_{\omega}\left(D^{2} w, D^{2} v\right), \quad \forall w \in \mathcal{W}^{\omega}, \forall v \in \mathcal{H}_{c}(\omega) .
$$

By the density of $\mathcal{W}^{\omega}$ in $D\left(G^{\omega}\right)$ combined with (2.19), the previous equality holds actually for any $u \in D\left(G^{\omega}\right)$. Therefore, we obtain the representation formula (2.16) for any open set $\omega$ with $\bar{\omega} \subset \Omega$.

Step 7. Consider an open set $\omega \subset \Omega$, and $u_{n}, u$ satisfying (2.5) and (2.11). Let us prove (2.13).

Let $O$ be an open set with $\bar{O} \subset \omega$. Since the space $\mathcal{W}^{O}$ of Step 6 is separable for the topology of $D\left(G^{O}\right)$, up to extract a subsequence, we may assume for any $w \in \mathcal{W}^{O}$, the convergence of the sequence

$$
\int_{O} A_{n} D^{2} u_{n}: D^{2} w_{n} d x
$$

where $w_{n}$ is defined by $(2.59)$, with $\omega=O$. We then define the linear mapping $f: \mathcal{W}^{O} \rightarrow \mathbb{R}$ by

$$
f(w)=\lim _{n \rightarrow \infty} \int_{O} A_{n} D^{2} u_{n}: D^{2} w_{n} d x, \quad \forall w \in \mathcal{W}^{O} .
$$

Since $w_{n}$ is a recovery sequence (for $G_{n}^{O}$ ) of limit $w$, and $A_{n} D^{2} u_{n}: D^{2} u_{n}$ is bounded in $L^{1}(\omega)$, the mapping $f$ is continuous in $\mathcal{W}^{O} \subset D\left(G^{O}\right)$. Therefore, we can extend $f$ to an element of $D\left(G^{O}\right)^{\prime}$, still denoted by $f$.

Let us now prove that for any $\zeta_{n}, \zeta \in H_{0}^{2}(O)$ such that $\zeta_{n}$ converges to $\zeta$ in $L^{2}(O)$ and $A_{n} D^{2} \zeta_{n}: D^{2} \zeta_{n}$ is bounded in $L^{1}(O)$, we have

$$
\int_{O} A_{n} D^{2} u_{n}: D^{2} \zeta_{n} d x \longrightarrow f(\zeta)
$$


To this end, consider a recovery sequence $\hat{\zeta}_{n}$ (for $G_{n}^{O}$ ) of limit $\zeta$, and a sequence $w^{k} \in \mathcal{W}^{O}$ which converges to $\zeta$ in $D\left(G^{O}\right)$. Also consider $w_{n}^{k}$ as the solution of $(2.59)$, where $w_{n}, w, \omega$ are replaced by $w_{n}^{k}, w^{k}, O$. We have

$$
\lim _{k \rightarrow \infty} \lim _{n \rightarrow \infty} \int_{O} A_{n} D^{2}\left(\hat{\zeta}_{n}-w_{n}^{k}\right): D^{2}\left(\hat{\zeta}_{n}-w_{n}^{k}\right) d x=\lim _{k \rightarrow \infty} G^{O}\left(\zeta-w^{k}\right)=0,
$$

which implies that

$$
\lim _{n \rightarrow \infty} \int_{O} A_{n} D^{2} u_{n}: D^{2} \hat{\zeta}_{n} d x=\lim _{k \rightarrow \infty} \lim _{n \rightarrow \infty} \int_{O} A_{n} D^{2} u_{n}: D^{2} w_{n}^{k} d x=\lim _{k \rightarrow \infty} f\left(w^{k}\right)=f(\zeta) .
$$

However, since $\hat{\zeta}_{n}-\zeta_{n}$ converges to zero in $L^{2}(\omega)$ and $A_{n} D^{2}\left(\hat{\zeta}_{n}-\zeta_{n}\right): D^{2}\left(\hat{\zeta}_{n}-\zeta_{n}\right)$ is bounded in $L^{1}(O)$, we have by $(2.11)$,

$$
\lim _{n \rightarrow \infty} \int_{O} A_{n} D^{2} u_{n}: D^{2}\left(\hat{\zeta}_{n}-\zeta_{n}\right) d x=0
$$

which yields (2.60) holds.

On the other hand, taking into account that $L^{2}(O)$ is dense in $D\left(G^{O}\right)^{\prime}$, there exists a sequence $h^{k}$ converging to $f$ in $D\left(G^{O}\right)^{\prime}$. Then, the solution $r^{k}$ of

$$
\left\{\begin{array}{l}
r^{k} \in D\left(G^{O}\right) \\
\Psi^{O}\left(r^{k}, \zeta\right)+\int_{O} r^{k} \zeta d x=\int_{O} h^{k} \zeta d x-f(\zeta), \quad \forall v \in D\left(G^{O}\right),
\end{array}\right.
$$

converges to zero in $D\left(G^{O}\right)$. Let $z_{n}^{k}$ be the solution of

$$
\left\{\begin{array}{l}
\operatorname{div}^{2}\left(A_{n} D^{2} z_{n}^{k}\right)+z_{n}^{k}=h^{k}+u_{n} \text { in } O \\
z_{n}^{k}-u_{n} \in H_{0}^{2}(O)
\end{array}\right.
$$

From $(2.11)$ and $(2.60)$, we deduce that $z_{n}^{k}-u_{n}$ is a recovery sequence (for $G_{n}^{O}$ ) of limit $r^{k}$. We thus have

$$
\lim _{k \rightarrow \infty} \lim _{n \rightarrow \infty} \int_{O} A_{n} D^{2}\left(z_{n}^{k}-u_{n}\right): D^{2}\left(z_{n}^{k}-u_{n}\right) d x=\lim _{k \rightarrow \infty} G^{O}\left(r^{k}\right)=0 .
$$

Now, consider $v_{n}$ and $v$ satisfying (2.14). Observe that by Step 5,

$$
A_{n} D^{2} z_{n}^{k}: D^{2} v_{n} \stackrel{*}{\longrightarrow} a_{O}\left(D^{2} z^{k}, D^{2} v\right) \text { in } \mathcal{M}(O)
$$

and by $(2.19)$,

$$
a\left(D^{2} z^{k}, D^{2} v\right) \stackrel{*}{\longrightarrow} a_{O}\left(D^{2} u, D^{2} v\right) \text { in } \mathcal{M}(O) .
$$

Finally, passing to the limit successively as $n \rightarrow \infty$ and $k \rightarrow \infty$ in

$$
A_{n} D^{2} u_{n}: D^{2} v_{n}=A_{n} D^{2}\left(u_{n}-z_{k}^{n}\right): D^{2} v_{n}+A_{n} D^{2} z_{k}^{n}: D^{2} v_{n}
$$

thanks to (2.63), (2.64) and (2.65), and taking into account (2.9), we obtain that

$$
A_{n} D^{2} u_{n}: D^{2} v_{n} \stackrel{*}{\longrightarrow} a_{O}\left(D^{2} u, D^{2} v\right)=a_{\omega}\left(D^{2} u, D^{2} v\right) \text { in } \mathcal{M}(O),
$$

for any open set $O$, with $\bar{O} \subset \omega$. This proves (2.13). 
Step 8. Let us prove that (2.16) holds for an arbitrary open set $\omega \subset \Omega$. To this end, note that the definition of $\Gamma$-convergence and Step 6 imply that for any $u \in \mathcal{H}_{c}(\omega)$, we have

$$
\begin{aligned}
G^{\omega}(u) & \leq \inf \left\{\begin{array}{l|l}
\liminf _{n \rightarrow \infty} \int_{\omega} A_{n} D^{2} u_{n}: D^{2} u_{n} d x: & \begin{array}{l}
u_{n} \rightarrow u \text { in } L^{2}(\omega) \\
\operatorname{supp}\left(u_{n}\right) \subset K \text { fixed compact of } \omega
\end{array}
\end{array}\right\} \\
& =\int_{\omega} d \mathfrak{a}_{\omega}\left(D^{2} u, D^{2} u\right) .
\end{aligned}
$$

Now, for $u \in \mathcal{H}_{c}(\omega)$, consider a recovery sequence $\hat{u}_{n}$ (for $G_{n}^{\omega}$ ) of limit $u$. For any $\varphi \in C_{0}^{0}(\omega)$, $0 \leq \varphi \leq 1$, we have thanks to $(2.13)$,

$$
G^{\omega}(u)=\lim _{n \rightarrow \infty} \int_{\omega} A_{n} D^{2} \hat{u}_{n}: D^{2} \hat{u}_{n} d x \geq \lim _{n \rightarrow \infty} \int_{\omega} A_{n} D^{2} \hat{u}_{n}: D^{2} \hat{u}_{n} \varphi d x=\int_{\omega} \varphi d \mathfrak{a}_{\omega}\left(D^{2} u, D^{2} u\right) .
$$

Hence, letting $\varphi$ increase to 1 , we get that

$$
G^{\omega}(u) \geq \int_{\omega} d \mathfrak{a}_{\omega}\left(D^{2} u, D^{2} u\right)
$$

which combined with (2.66) yields the representation formula (2.16) for $G^{\omega}$.

Step 9. In order to conclude the proof of theorem 2.5, it remains to show (2.17) and (2.18). To this end, for a given $u \in \mathcal{H}(\omega)$, consider a sequence $u_{n}$ converging to $u$ which satisfies $(2.11)$ (for example, up to extract a subsequence, take a recovery sequence of limit $u$ for the sequence $F_{n}^{\omega}$ defined by (2.47)). Then, for two open sets $O, U$, with $\bar{O} \subset U \subset \bar{U} \subset \omega$, consider a function $\varphi \in C_{0}^{0}(\omega)$ such that $0 \leq \varphi \leq 1$ in $\omega$, and $\varphi \equiv 1$ in $U$. Then, passing to the limit in the inequality

$$
\begin{aligned}
\alpha(O, U) \int_{O}\left|D^{2} u_{n}\right|^{2} d x & \leq \int_{U}\left|u_{n}\right|^{2} d x+\int_{U} A_{n} D^{2} u_{n}: D^{2} u_{n} d x \\
& \leq \int_{\omega}\left|u_{n}\right|^{2} \varphi d x+\int_{\omega} A_{n} D^{2} u_{n}: D^{2} u_{n} \varphi d x
\end{aligned}
$$

we deduce from the lower semi-continuity for the $L^{2}(O)$ norm of $D^{2} u_{n}$ and from convergence (2.13), that

$$
\alpha(O, U) \int_{O}\left|D^{2} u\right|^{2} d x \leq \int_{\omega}|u|^{2} \varphi d x+\int_{\omega} \varphi d \mathfrak{a}\left(D^{2} u, D^{2} u\right) \leq \int_{\omega}|u|^{2} d x+\int_{\omega} d \mathfrak{a}\left(D^{2} u, D^{2} u\right),
$$

which establishes (2.17).

On the other hand, let $\varphi \in C_{0}^{0}(\omega), 0 \leq \varphi \leq 1$. If in addition $u \in W^{2, \infty}(\omega)$, then we have again by (2.13) combined with the Cauchy-Schwarz inequality and (2.1),

$$
\begin{aligned}
\int_{\omega} \varphi d \mathfrak{a}_{\omega}\left(D^{2} u, D^{2} u\right) & =\lim _{n \rightarrow \infty} \int_{\omega} A_{n} D^{2} u_{n}: D^{2} u \varphi d x \\
& \leq \limsup _{n \rightarrow \infty}\left(\int_{\omega} A_{n} D^{2} u_{n}: D^{2} u_{n} \varphi d x\right)^{\frac{1}{2}}\left(\int_{\omega} A_{n} D^{2} u: D^{2} u \varphi d x\right)^{\frac{1}{2}} \\
& \leq\left(\int_{\omega} \varphi d \mathfrak{a}_{\omega}\left(D^{2} u, D^{2} u\right)\right)^{\frac{1}{2}}\left(\int_{\omega}\left|D^{2} u\right|^{2} \varphi d \mu\right)^{\frac{1}{2}} \\
& \leq\left(\int_{\omega} \varphi d \mathfrak{a}_{\omega}\left(D^{2} u, D^{2} u\right)\right)^{\frac{1}{2}}\left(\mu(\omega)\left\|D^{2} u\right\|_{L^{\infty}\left(\omega ; \mathbb{R}_{s}^{2 \times 2}\right)}^{2}\right)^{\frac{1}{2}}
\end{aligned}
$$


This yields the desired bound (2.18) by letting $\varphi$ converge to 1 .

Proof of Theorem 2.6. For an open set $\omega \subset \Omega$, up to a subsequence, the functional $F_{n}^{\omega}$ defined by (2.47) $\Gamma$-converges to some $F^{\omega}$. Let $u \in \mathcal{H}(\omega)$, and consider a recovery sequence $u_{n}$ (for $F_{n}^{\omega}$ ) of limit $u$. Then, we know by (2.13) (applied to a quadratic function $v_{n}=v$ ) that for any $\xi \in \mathbb{R}_{s}^{2 \times 2}$, the following limit holds

$$
A_{n} D^{2} u_{n}: \xi \stackrel{*}{\longrightarrow} \mathfrak{a}_{\omega}\left(D^{2} u, \xi\right) \text { in } \mathcal{M}(\omega) .
$$

This implies that

$$
A_{n} D^{2} u_{n} \stackrel{*}{\longrightarrow} \sigma \text { in } \mathcal{M}\left(\omega ; \mathbb{R}_{s}^{2 \times 2}\right), \quad \text { with } \sigma: \xi=\mathfrak{a}_{\omega}\left(D^{2} u, \xi\right), \quad \forall \xi \in \mathbb{R}_{s}^{2 \times 2} .
$$

By (2.67) and (2.13), we also have that for any $v \in \mathcal{H}(\omega) \cap C^{2}(\omega)$ and for any $\varphi \in C_{c}^{0}(\omega)$,

$$
\int_{\omega} \varphi D^{2} v: d \sigma=\lim _{n \rightarrow \infty} \int_{\omega} A_{n} D^{2} u_{n}: D^{2} v \varphi d x=\int_{\omega} \varphi d \mathfrak{a}_{\omega}\left(D^{2} u, D^{2} v\right),
$$

which gives

$$
\sigma: D^{2} v=\mathfrak{a}_{\omega}\left(D^{2} u, D^{2} v\right) \text { in } \omega .
$$

On the other hand we have for any $\varphi \in C_{0}^{0}(\omega), \varphi \geq 0$, and for any $\xi \in \mathbb{R}_{s}^{2 \times 2}$,

$$
\left|\int_{\omega} A_{n} D^{2} u_{n}: \xi \varphi d x\right| \leq\left(\int_{\omega} A_{n} D^{2} u_{n}: D^{2} u_{n} \varphi d x\right)^{\frac{1}{2}}\left(\int_{\omega} A_{n} \xi: \xi \varphi d x\right)^{\frac{1}{2}} .
$$

Taking into account (2.1) and

$$
A_{n} D^{2} u_{n}: D^{2} u_{n} \stackrel{*}{\longrightarrow} \mathfrak{a}_{\omega}\left(D^{2} u, D^{2} u\right) \text { in } \mathcal{M}\left(\omega ; \mathbb{R}_{s}^{2 \times 2}\right)
$$

which is a consequence of (2.13), we can pass to the limit as $n \rightarrow \infty$ in the above inequality to deduce that

$$
\left|\int_{\omega} \varphi \xi: d \sigma\right| \leq\left(\int_{\omega} \varphi d \mathfrak{a}\left(D^{2} u, D^{2} u\right)\right)^{\frac{1}{2}}\left(\int_{\omega} \varphi d \mu\right)^{\frac{1}{2}}|\xi| .
$$

This proves that $\sigma$ is absolutely continuous with respect to $\mu$. Therefore, by the Radon-Nikodym theorem there exists a function $\mathcal{A}_{\omega} D^{2} u \in L_{\mu}^{1}\left(\omega ; \mathbb{R}_{s}^{2 \times 2}\right)$ such that $\sigma=\mathcal{A}_{\omega} D^{2} u \mu$, which satisfies (2.22). Since

$$
\mathcal{A}_{\omega} D^{2} u: \xi \mu=\mathfrak{a}_{\omega}\left(D^{2} u, \xi\right), \quad \forall \xi \in \mathbb{R}_{s}^{2 \times 2},
$$

the operator $\mathcal{A}_{\omega}$ is linear with respect to $D^{2} u$, and by (2.68) satisfies (2.21). Moreover, $\mathcal{A}_{\omega}$ inherits the strong locality property of $a_{\omega}$.

By the proof of Theorem 2.5, for any ball $B_{m}$ of the countable basis of the topology of $\Omega$ used to define $\mathfrak{a}_{\omega}, \mathfrak{a}_{\omega}\left(D^{2} u, D^{2} u\right)$ is the weak-* limit in $\mathcal{M}\left(B_{m}\right)$ of a sequence $\mathfrak{a}_{\omega}\left(D^{2} u^{k}, D^{2} u^{k}\right)$, where $u^{k}$ is the limit of a recovery sequence $u_{n}^{k}$ for $F_{n}^{B_{m}}$ such that $\operatorname{div}^{2}\left(A_{n} D^{2} u_{n}^{k}\right)$ is compact in $H^{-2}\left(\omega ; \mathbb{R}_{s}^{2 \times 2}\right)$. Then, by virtue of Lemma 2.13 the sequence $A_{n} D^{2} u_{n}^{k}$ is bounded in $H^{-1}\left(\omega ; \mathbb{R}_{s}^{2 \times 2}\right)$, so that $\mathcal{A}_{\omega} D^{2} u^{k} \mu$ belongs to $H^{-1}\left(\omega ; \mathbb{R}_{s}^{2 \times 2}\right)$. Therefore, $\mathcal{A}_{\omega} D^{2} u^{k} \mu$ vanishes on sets of zero capacity. Moreover, $\mathcal{A}_{\omega} D^{2} u$ is the strong limit of $\mathcal{A}_{\omega} D^{2} u^{k}$ in $L_{\mu}^{1}\left(\omega ; \mathbb{R}_{s}^{2 \times 2}\right)$, hence it vanishes on sets of zero capacity.

We now define $A \in L^{1}\left(\Omega ; \mathcal{L}_{s}\left(\mathbb{R}_{s}^{2 \times 2}\right)\right)$ by

$$
A \xi=\mathcal{A}_{\Omega} \xi \quad \forall \xi \in \mathcal{M}\left(\omega ; \mathbb{R}_{s}^{2 \times 2}\right), \mu \text {-a.e. in } \Omega \text {. }
$$


Let $u \in C^{2}(\omega) \cap \mathcal{H}(\omega)$, let $B\left(x_{0}, r+\delta\right)$ be a ball of $\omega$ with $r, \delta>0$, and let $\varphi \in C_{c}^{0}\left(B\left(x_{0}, r+\delta\right)\right.$ with $\varphi \equiv 1$ in $B\left(x_{0}, r\right), 0 \leq \varphi \leq 1$ in $B\left(x_{0}, r+\delta\right)$. Consider a recovery sequence $u_{n}$ for $F_{n}^{B\left(x_{0}, r+\delta\right)}$ of limit $u$. By (2.67), (2.68), (2.70) and applying (2.69), with $\omega=B\left(x_{0}, r+\delta\right)$, we get for any $\xi \in \mathbb{R}_{s}^{2 \times 2}$,

$$
\begin{aligned}
& \left|\int_{B\left(x_{0}, r+\delta\right)} \mathcal{A}_{B\left(x_{0}, r+\delta\right)} D^{2} u: \xi \varphi d \mu\right|=\lim _{n \rightarrow \infty}\left|\int_{B\left(x_{0}, r+\delta\right)} A_{n} D^{2} u_{n}: \xi \varphi d x\right| \\
& \leq \lim _{n \rightarrow \infty}\left(\int_{B\left(x_{0}, r+\delta\right)} A_{n} D^{2} u_{n}: D^{2} u_{n} \varphi d x\right)^{\frac{1}{2}}\left(\int_{B\left(x_{0}, r+\delta\right)} A_{n} \xi: \xi \varphi d x\right)^{\frac{1}{2}} \\
& \leq \lim _{n \rightarrow \infty}\left(\int_{B\left(x_{0}, r+\delta\right)} A_{n} D^{2} u_{n}: D^{2} u_{n} d x\right)^{\frac{1}{2}}\left(\int_{B\left(x_{0}, r+\delta\right)} A_{n} \xi: \xi \varphi d x\right)^{\frac{1}{2}} \\
& \leq \lim _{n \rightarrow \infty}\left(\int_{B\left(x_{0}, r+\delta\right)} A_{n} D^{2} u: D^{2} u d x\right)^{\frac{1}{2}}\left(\int_{B\left(x_{0}, r+\delta\right)} A_{n} \xi: \xi \varphi d x\right)^{\frac{1}{2}} \\
& \leq\left(\int_{\bar{B}\left(x_{0}, r+\delta\right)}\left|D^{2} u\right|^{2} d \mu\right)^{\frac{1}{2}} \mu\left(\bar{B}\left(x_{0}, r+\delta\right)\right)^{\frac{1}{2}}|\xi| .
\end{aligned}
$$

Then, letting $\varphi$ converge to 1 and $\delta$ converge to 0 , and noting that $\mathcal{A}_{B\left(x_{0}, r+\delta\right)} D^{2} u=\mathcal{A}_{\omega} D^{2} u$ (due to the strong locality property), we get

$$
\left|\int_{\bar{B}\left(x_{0}, r\right)} \mathcal{A}_{\omega} D^{2} u: \xi d \mu\right| \leq\left(\int_{\bar{B}\left(x_{0}, r\right)}\left|D^{2} u\right|^{2} d \mu\right)^{\frac{1}{2}} \mu\left(\bar{B}\left(x_{0}, r\right)\right)^{\frac{1}{2}}|\xi|,
$$

for any $\xi \in \mathbb{R}^{N}$ and for any $\bar{B}\left(x_{0}, \delta\right) \subset \omega$. Using the derivation measures theorem and the local property (2.20) of the operator $\mathcal{A}_{\omega}$, the previous inequality implies that

$$
\left|\mathcal{A}_{\omega} D^{2} u\right| \leq\left|D^{2} u\right| \quad \mu \text {-a.e. in } \omega \text {. }
$$

Applying (2.72) to the function $x \mapsto u-\frac{1}{2} D^{2} u\left(x_{0}\right) x \cdot x$, and taking into account the definition of $A$, we also have

$$
\left|\mathcal{A}_{\omega} D^{2} u-A D^{2} u\left(x_{0}\right)\right| \leq\left|D^{2} u-D^{2} u\left(x_{0}\right)\right| \quad \mu \text {-a.e. in } \omega .
$$

Therefore, due to the continuity of $D^{2} u$ this yields (2.24).

Finally, inequality (2.23) is a simple consequence of (2.72), where $\omega=\Omega$ and $u$ is a quadratic function.

Proof of Proposition 2.8. Let $\omega$ be an open of $\Omega$, and let $O^{1}, O^{2}$ be two disjoint open sets of class $C^{2}$ such that $(2.26)$ holds. Let $u$ be a function in $C^{1}(\bar{\omega})$, with $u \in C^{2}\left(\overline{\omega \cap O^{1}}\right) \cap C^{2}\left(\overline{\omega \cap O^{2}}\right)$. Let us obtain a representation of $\mathfrak{a}_{\omega}\left(D^{2} u, D^{2} u\right)$. Thanks to the strong locality property (2.9) of $\mathfrak{a}_{\omega}$, it is enough to derive for any $x_{0} \in \omega$, a neighborhood $O$ of $x_{0}$, for which we can construct a representation formula for $\mathfrak{a}_{O}\left(D^{2} u, D^{2} u\right)$.

If $x_{0} \in O^{1} \cup O^{2}$, then taking $O=O^{1} \cup O^{2}$, we have

$$
\mathfrak{a}_{O^{1} \cup O^{2}}\left(D^{2} u, D^{2} u\right)=A D^{2} u: D^{2} u \mu \text { in } O^{1} \cup O^{2},
$$

using that $u \in C^{2}\left(O^{1} \cup O^{2}\right) \cap \mathcal{H}\left(O^{1} \cup O^{2}\right)$ and the representations (2.21), (2.24) of Theorem 2.6.

If $x_{0} \in \partial O^{1} \cap \omega$, then there exist $r>0$, an open neighborhood $O$ of $x_{0}$, and a $C^{2}$ diffeomorphism from $B(0, r)$ onto $O$, such that

$$
\psi(0)=x_{0}, \quad \psi(t, s) \in O^{1} \Longleftrightarrow s>0, \quad \psi(t, s) \in \partial O^{1} \Longleftrightarrow s=0 .
$$


Denote

$$
\begin{gathered}
B(0, r)^{+}=B(0, r) \cap(\mathbb{R} \times(0, \infty)), \quad \tilde{\gamma}=B(0, r) \cap(\mathbb{R} \times\{0\}), \quad \tilde{B}(0, r)^{+}=B(0, r)^{+} \cup \tilde{\gamma} \\
O^{+}=\psi\left(B(0, r)^{+}\right), \quad \gamma=\psi(\tilde{\gamma}) .
\end{gathered}
$$

Define $\tilde{u}=u \circ \psi \in C^{1}(\bar{B}(0, r)), \tilde{u}^{+} \in C^{2}\left(\tilde{B}(0, r)^{+}\right)$as the restriction of $\tilde{u}$ to $\tilde{B}(0, r)^{+}$, and $u^{+}=\tilde{u}^{+} \circ \psi^{-1} \in C^{2}\left(\overline{O^{+}}\right)$. Taking $\mathrm{n}=\nabla\left(\psi^{-1}\right)_{2}$ in $\gamma$, which is a normal vector to $\gamma$, let us prove that

$$
\begin{aligned}
& \mathfrak{a}_{O}\left(D^{2} u, D^{2} u\right)=\chi_{O \backslash \gamma} A D^{2} u: D^{2} u \mu \\
& +\chi_{\gamma} A\left(D^{2} u^{+}-\left(\frac{\partial^{2} \tilde{u}^{+}}{\partial s^{2}} \circ \psi^{-1}\right) \mathrm{n} \odot \mathrm{n}\right):\left(D^{2} u^{+}-\left(\frac{\partial^{2} \tilde{u}^{+}}{\partial s^{2}} \circ \psi^{-1}\right) \mathrm{n} \odot \mathrm{n}\right) \mu .
\end{aligned}
$$

To this end, consider for $\eta \in C^{\infty}(\mathbb{R})$ such that $\theta(s)=1$ if $|s|>2, \theta(s)=0$ if $|s|<1$, the function $\tilde{u}_{\varepsilon} \in C^{2}(\bar{B}(0, r)), \varepsilon>0$, defined by

$$
\tilde{u}_{\varepsilon}(t, s)=\tilde{u}(t, s) \theta\left(\frac{s}{\varepsilon}\right)+\left(\tilde{u}(t, 0)+\frac{\tilde{u}(t, \varepsilon)-\tilde{u}(t,-\varepsilon)}{2 \varepsilon} s\right)\left(1-\theta\left(\frac{s}{\varepsilon}\right)\right),
$$

and set $u_{\varepsilon}=\tilde{u}_{\varepsilon} \circ \psi^{-1} \in C^{2}(\bar{O})$. Then, by (2.24) we have

$$
\mathfrak{a}_{O}\left(D^{2} u_{\varepsilon}, D^{2} u_{\varepsilon}\right)=A D^{2} u_{\varepsilon}: D^{2} u_{\varepsilon} \mu \text { in } O .
$$

Moreover, we can check that $D^{2} u_{\varepsilon}$ is bounded in $L^{\infty}\left(O, \mathbb{R}_{s}^{2 \times 2}\right)$ and

$$
D^{2} u_{\varepsilon} \longrightarrow \Lambda=D^{2} u-\chi_{\gamma}\left(\frac{\partial^{2} \tilde{u}^{+}}{\partial s^{2}} \circ \psi^{-1}\right) \mathrm{n} \odot \mathrm{n} \quad \text { pointwise in } O .
$$

Then, by the Lebesgue dominated convergence theorem we get that

$$
A\left(D^{2} u_{\varepsilon}-\Lambda\right):\left(D^{2} u_{\varepsilon}-\Lambda\right) \longrightarrow 0 \text { in } L_{\mu}^{1}(O) .
$$

The previous convergence and (2.21) imply that $u_{\varepsilon}$ satisfies in $O$ the Cauchy property (2.78) of the following

Lemma 2.15. Let $\omega$ be an open set of $\Omega$. Consider $u_{\varepsilon}, u \in \mathcal{H}(\omega)$ such that $u_{\varepsilon}$ converges to $u$ in $L^{2}(\omega)$ and

$$
\forall \rho>0, \exists \tau>0, \forall \varepsilon, \delta \in(0, \tau), \quad \int_{\omega} d \mathfrak{a}_{\omega}\left(D^{2}\left(u_{\varepsilon}-u_{\delta}\right): D^{2}\left(u_{\varepsilon}-u_{\delta}\right)\right)<\rho .
$$

Then, the sequence $\mathfrak{a}_{\omega}\left(D^{2} u_{\varepsilon}: D^{2} u_{\varepsilon}\right)$ converges strongly to $\mathfrak{a}_{\omega}\left(D^{2} u: D^{2} u\right)$ in $\mathcal{M}(\omega)$.

Therefore, by virtue of Lemma 2.15 the sequence $\mathfrak{a}_{O}\left(D^{2} u_{\varepsilon}, D^{2} u_{\varepsilon}\right)$ converges strongly to $\mathfrak{a}_{O}\left(D^{2} u, D^{2} u\right)$ in $\mathcal{M}(O)$. This combined with (2.75) and (2.77) yields (2.74).

For any $v \in C^{2}(\bar{\omega})$, applying (2.74) to $u+v$ and $u-v$, we also obtain that

$$
\mathfrak{a}_{0}\left(D^{2} u, D^{2} v\right)=\chi_{O \backslash \gamma} A D^{2} u: D^{2} v \mu+\chi_{\gamma} A\left(D^{2} u^{+}-\left(\frac{\partial^{2} \tilde{u}^{+}}{\partial^{2} s^{2}} \circ \psi^{-1}\right) \mathrm{n} \odot \mathrm{n}\right): D^{2} v \mu,
$$

in a neighborhood $O$ of any point $x_{0}$ of $\partial O^{1} \cap \partial O^{2}$.

Now, consider an open curve $\gamma$ of class $C^{2}$ given as the range of a function $\eta \in C^{2}\left((0,1) ; \mathbb{R}^{2}\right)$ such that $\eta^{\prime}(t) \neq 0$, for any $t \in(0,1)$. For $x_{0}=\eta\left(t_{0}\right) \in \gamma$, consider a vector $\zeta$ linearly 
independent with $\eta^{\prime}\left(t_{0}\right)$. Then, by the inverse function theorem there exists a ball $B(0, r)$ and a neighborhood $O$ of $x_{0}$ such that the function $\psi: B(0, r) \rightarrow O$ defined by

$$
\psi(t, s)=\eta\left(t_{0}+t\right)+s \zeta, \quad \forall(t, s) \in B(0, r)
$$

is $C^{2}$-diffeomorphism from $B(0, r)$ onto $O$. Consider for $\delta>0$, the function $\tilde{u}_{\delta} \in C^{1}(B(0, r))$ defined by

$$
\tilde{u}_{\delta}(t, s)= \begin{cases}s^{2} & \text { if } s \geq 0 \\ (1-\delta) s^{2} & \text { if } s \leq 0\end{cases}
$$

and set $u_{\delta}=\tilde{u}_{\delta} \circ \psi^{-1} \in C^{1}(O)$. Defining $u=\tilde{u}_{0} \circ \psi^{-1}$, and using (2.74) and (2.79) we have

$$
\begin{aligned}
& \mathfrak{a}_{O}\left(D^{2} u_{\delta}, D^{2} u_{\delta}\right)=\chi_{O \backslash \gamma} A D^{2} u_{\delta}: D^{2} u_{\delta} \mu+\chi_{\gamma \cap O} A\left(D^{2} u-2 \mathrm{n} \odot \mathrm{n}\right):\left(D^{2} u-2 \mathrm{n} \odot \mathrm{n}\right) \mu \\
& \mathfrak{a}_{O}\left(D^{2} u_{\delta}, D^{2} u\right)=\chi_{O \backslash \gamma} A D^{2} u_{\delta}: D^{2} u \mu+\chi_{\gamma \cap O} A\left(D^{2} u-2 \mathrm{n} \odot \mathrm{n}\right): D^{2} u \mu,
\end{aligned}
$$

where $\mathrm{n}$ is the normal vector to $\gamma \cap O$ given by

$$
\mathrm{n}(\psi(t, 0))=\frac{J \eta^{\prime}\left(t_{0}+t\right)}{J \eta^{\prime}\left(t_{0}+t\right) \cdot \zeta}
$$

Clearly, $\tilde{u}_{\delta}$ converges to $\tilde{u}_{0}(t, s)$ in $W^{2, \infty}(B(0, r))$. Then, $u_{\delta}$ converges to $u$ in $W^{2, \infty}(O)$. This combined with (2.18) implies that $\mathfrak{a}_{O}\left(D^{2} u_{\delta}, D^{2} u_{\delta}\right)$ and $\mathfrak{a}_{O}\left(D^{2} u_{\delta}, D^{2} u\right)$ converge strongly to $\mathfrak{a}_{O}\left(D^{2} u, D^{2} u\right)$ in $\mathcal{M}(O)$. On the other hand, passing to the limit in (2.80) we obtain

$$
\begin{aligned}
& \chi_{O \backslash \gamma} A D^{2} u_{\delta}: D^{2} u_{\delta}+\chi_{\gamma \cap O} A\left(D^{2} u-2 \mathrm{n} \odot \mathrm{n}\right):\left(D^{2} u-2 \mathrm{n} \odot \mathrm{n}\right) \\
& \longrightarrow \chi_{O \backslash \gamma} A D^{2} u: D^{2} u+\chi_{\gamma \cap O} A\left(D^{2} u-2 \mathrm{n} \odot \mathrm{n}\right):\left(D^{2} u-2 \mathrm{n} \odot \mathrm{n}\right) \text { in } L_{\mu}^{1}(O), \\
& \chi_{O \backslash \gamma} A D^{2} u_{\delta}: D^{2} u+\chi_{\gamma \cap O} A\left(D^{2} u-2 \mathrm{n} \odot \mathrm{n}\right): D^{2} u \\
& \longrightarrow \chi_{O \backslash \gamma} A D^{2} u: D^{2} u+\chi_{\gamma \cap O} A\left(D^{2} u-2 \mathrm{n} \odot \mathrm{n}\right): D^{2} u \text { in } L_{\mu}^{1}(O),
\end{aligned}
$$

Moreover, since $u \in C^{2}(O)$, we also have

$$
\mathfrak{a}_{\omega}\left(D^{2} u, D^{2} u\right)=A D^{2} u: D^{2} u \mu \text { in } O .
$$

Hence, from the three previous expressions involving $\mathfrak{a}_{\omega}\left(D^{2} u, D^{2} u\right)$, we deduce that $A\left(D^{2} u-2 \mathrm{n} \odot \mathrm{n}\right):\left(D^{2} u-2 \mathrm{n} \odot \mathrm{n}\right)=A\left(D^{2} u-2 \mathrm{n} \odot \mathrm{n}\right): D^{2} u=A D^{2} u: D^{2} u \quad \mu$-a.e. in $\gamma \cap O$, which implies that

$$
A(\mathrm{n} \odot \mathrm{n}):(\mathrm{n} \odot \mathrm{n})=0 \quad \mu \text {-a.e. in } \gamma \cap O .
$$

Since this holds for any $x \in \gamma$, we get (2.25).

Finally, taking into account (2.25) in (2.74), formula (2.74) can be written as

$$
\mathfrak{a}_{O}\left(D^{2} u, D^{2} u\right)=A D^{2} u: D^{2} u \mu \text { in } O,
$$

which combined with (2.73) yields

$$
\mathfrak{a}_{\omega}\left(D^{2} u, D^{2} u\right)=A D^{2} u: D^{2} u \mu \text { in } \omega, \quad \forall u \in C^{1}(\bar{\omega}), u \in C^{2}\left(\overline{\omega \cap O^{1}}\right) \cap C^{2}\left(\overline{\omega \cap O^{2}}\right) .
$$

Therefore, equality (2.24) holds for any $u \in C^{1}(\bar{\omega})$, with $u \in C^{2}\left(\overline{\omega \cap O^{1}}\right) \cap C^{2}\left(\overline{\omega \cap O^{2}}\right)$. 
Proof of Lemma 2.15. First, let us prove the following semi-continuity result: For any $v_{\varepsilon}, v \in \mathcal{H}(\omega)$ such that $v_{\varepsilon}$ converges to $v$ in $L^{2}(\omega)$, we have

$$
\int_{\omega} d \mathfrak{a}_{\omega}\left(D^{2} v, D^{2} v\right) \leq \liminf _{\varepsilon \rightarrow \infty} \int_{\omega} d \mathfrak{a}_{\omega}\left(D^{2} v_{\varepsilon}, D^{2} v_{\varepsilon}\right) .
$$

To prove this we can assume that the right-hand side is finite. Let $\varphi \in C_{0}^{0}(\omega)$, with $0 \leq \varphi \leq 1$ on $\omega$. By (2.19) we have

$$
\begin{aligned}
0 & \leq \liminf _{\varepsilon \rightarrow 0} \int_{\omega} \varphi d \mathfrak{a}_{\omega}\left(D^{2}\left(v_{\varepsilon}-v\right), D^{2}\left(v_{\varepsilon}-v\right)\right) \\
& =\liminf _{\varepsilon \rightarrow 0} \int_{\omega} \varphi d \mathfrak{a}_{\omega}\left(D^{2} v_{\varepsilon}, D^{2} v_{\varepsilon}\right)-2 \lim _{\varepsilon \rightarrow 0} \int_{\omega} \varphi d \mathfrak{a}_{\omega}\left(D^{2} v_{\varepsilon}, D^{2} v\right)+\int_{\omega} \varphi d \mathfrak{a}_{\omega}\left(D^{2} v, D^{2} v\right) \\
& \leq \liminf _{\varepsilon \rightarrow 0} \int_{\omega} d \mathfrak{a}_{\omega}\left(D^{2} v_{\varepsilon}, D^{2} v_{\varepsilon}\right)-\int_{\omega} \varphi d \mathfrak{a}_{\omega}\left(D^{2} v, D^{2} v\right) .
\end{aligned}
$$

Now, letting $\varphi$ increase to 1 we get (2.82).

On the other hand, applying (2.82) to $v_{\varepsilon}=u_{\varepsilon}-u_{\delta}$ with a fixed $\delta$, we deduce from (2.78) that for any $\rho>0$, there exists $\tau>0$, such that

$$
\int_{\omega} d \mathfrak{a}_{\omega}\left(D^{2}\left(u_{\delta}-u\right), D^{2}\left(u_{\delta}-u\right)\right) \leq \rho, \quad \forall \delta \in(0, \tau),
$$

which implies that

$$
\lim _{\varepsilon \rightarrow 0} \int_{\omega} d \mathfrak{a}_{\omega}\left(D^{2}\left(u_{\varepsilon}-u\right), D^{2}\left(u_{\varepsilon}-u\right)\right)=0 .
$$

Using this limit, the boundedness of a Cauchy sequence, and the following inequality

$$
\begin{aligned}
& \left\|a_{\omega}\left(D^{2} u_{\varepsilon}, D^{2} u_{\varepsilon}\right)-a_{\omega}\left(D^{2} u, D^{2} u\right)\right\|_{\mathcal{M}(\omega)} \\
& =\sup _{\|\varphi\|_{C_{0}^{0}(\omega)} \leq 1}\left|\int_{\omega} \varphi d \mathfrak{a}_{\omega}\left(D^{2} u_{\varepsilon}, D^{2} u_{\varepsilon}\right)-\int_{\omega} \varphi d \mathfrak{a}_{\omega}\left(D^{2} u, D^{2} u\right)\right| \\
& =\sup _{\|\varphi\|_{C_{0}^{0}(\omega)} \leq 1}\left|\int_{\omega} \varphi d \mathfrak{a}_{\omega}\left(D^{2}\left(u_{\varepsilon}+u\right), D^{2}\left(u_{\varepsilon}-u\right)\right)\right| \\
& \leq\left(\int_{\omega} d \mathfrak{a}_{\omega}\left(D^{2}\left(u_{\varepsilon}+u\right), D^{2}\left(u_{\varepsilon}+u\right)\right)\right)^{\frac{1}{2}}\left(\int_{\omega} d \mathfrak{a}_{\omega}\left(D^{2}\left(u_{\varepsilon}-u\right), D^{2}\left(u_{\varepsilon}-u\right)\right)\right)^{\frac{1}{2}},
\end{aligned}
$$

we thus conclude that $a_{\omega}\left(D^{2} u_{\varepsilon}, D^{2} u_{\varepsilon}\right)$ converges to $a_{\omega}\left(D^{2} u, D^{2} u\right)$ in $\mathcal{M}(\omega)$.

\section{Application to the homogenization of high-viscosity Stokes equations}

\subsection{Statement of the results}

In this section we study the homogenization of two-dimensional high-viscosity Stokes equations. We could prove independently of Section 2 similar results to Theorem 2.5 and Theorem 2.6 by adapting the div-curl result of Theorem 2.11 to the Stokes problem. For the sake of simplicity we have preferred applying the results of Section 2 by using the classical representation of a 
divergence free function as the rotated gradient of a stream function (see, e.g., [19]). This representation allows us to transform a Stokes equation into a simply connected domain by a fourth-order plate equation.

Consider a bounded domain $\Omega$ of $\mathbb{R}^{2}$ and a sequence $B_{n} \in L^{\infty}\left(\Omega ; \mathcal{L}_{s}\left(\mathbb{R}_{0}^{2 \times 2}\right)\right)$ such that

$$
\begin{gathered}
\left|B_{n}\right| \stackrel{*}{\longrightarrow} \nu \quad \text { in } \mathcal{M}(\Omega), \\
B_{n} \xi: \xi \geq 0, \quad \forall \xi \in \mathbb{R}_{0}^{2 \times 2}, \text { a.e. in } \Omega,
\end{gathered}
$$

and such that for any open sets $O, \omega$ with $\bar{O} \subset \omega \subset \Omega$, there exists a constant $\beta(O, \omega)$ satisfying

$$
\beta(O, \omega) \int_{O}|D u|^{2} d x \leq \int_{\omega}|u|^{2} d x+\int_{\omega} B_{n} D u: D u d x, \quad \forall u \in H_{\mathrm{div}}^{1}(\omega) .
$$

Our strategy here is to use the results of Section 2 to study the asymptotic behavior of a sequence of Stokes problems with viscosity matrix $B_{n}$, i.e.

$$
\left\{\begin{array}{l}
-\operatorname{Div}\left(B_{n} D u_{n}\right)+\nabla p_{n}=g_{n} \text { in } \omega \\
\operatorname{div} u_{n}=0 \text { in } \omega,
\end{array}\right.
$$

where $\omega$ is an open subset of $\Omega$ and $g_{n}$ is a compact sequence in $H^{-1}\left(\omega ; \mathbb{R}_{0}^{2 \times 2}\right)$. The functions $u_{n}$ and $p_{n}$ represent respectively the velocity and the pressure of the fluid. Similarly to the case of the plate equations we assume that the sequence $u_{n}$ has a bounded energy, i.e.

$$
\limsup _{n \rightarrow \infty}\left(\int_{\omega} B_{n} D u_{n}: D u_{n} d x+\int_{\omega}\left|u_{n}\right|^{2} d x\right)<\infty
$$

Remark 3.1. In order to study the homogenization of (3.4), we introduce the sequence $A_{n} \in$ $L^{\infty}\left(\Omega ; \mathcal{L}_{s}\left(\mathbb{R}_{s}^{2}\right)\right)$ defined by

$$
A_{n} \xi=-J B_{n}(J \xi), \quad \forall \xi \in \mathbb{R}_{s}^{2 \times 2}, \text { a.e. } x \in \Omega .
$$

Observe that if $\omega$ is a Lipschitz simply connected open subset of $\Omega$ and $u_{n} \in H_{\text {div }}^{1}(\omega)$ (see, e.g., [19]) there exists $w_{n} \in H^{2}(\omega)$ with zero mean value in $\omega$ such that $u_{n}=J \nabla w_{n}$. Moreover, if $u_{n}$ satisfies (3.4), (3.5), then the sequence $w_{n}$ satisfies $D u_{n}=J D^{2} w_{n}$ and $B_{n} D u_{n}=J A_{n} D^{2} w_{n}$. Hence, we deduce that

$$
\begin{gathered}
\operatorname{div}^{2}\left(A_{n} D^{2} w_{n}\right)=\operatorname{div}\left(J g_{n}\right) \text { in } \omega \\
\limsup _{n \rightarrow \infty}\left(\int_{\omega} A_{n} D^{2} w_{n}: D^{2} w_{n} d x+\int_{\omega}\left|w_{n}\right|^{2} d x\right) \\
\leq \limsup _{n \rightarrow \infty}\left(\int_{\omega} B_{n} D u_{n}: D u_{n} d x+C \int_{\omega}\left|u_{n}\right|^{2} d x\right)<\infty,
\end{gathered}
$$

where the last inequality is a consequence of the Poincaré-Wirtinger inequality in $\omega$. This permits to apply the results of Section 2 to the sequence $w_{n}$.

Remark 3.2. Combining Remark 2.2 and Remark 3.1, a sufficient condition to have (3.3) is to assume the existence of a positive number $\alpha$ and a sequence $E_{n}$ in $C^{0}\left(\Omega ; \mathbb{R}_{0}^{2 \times 2}\right)$, with $J E_{n} \geq \alpha I$ (note that $J E_{n}$ is symmetric since $E_{n}$ has zero trace), such that $B_{n}$ satisfies

$$
B_{n}(x) \xi: \xi \geq\left|E_{n}(x): \xi\right|^{2}, \quad \forall \xi \in \mathbb{R}_{s}^{2 \times 2} \text {, a.e. } x \in \omega, \forall n \in \mathbb{N} \text {. }
$$


Remark 3.3. From a physical point of view, the Stokes problem is usually written as

$$
\left\{\begin{array}{l}
-\operatorname{Div}\left(C_{n} e\left(u_{n}\right)\right)+\nabla p_{n}=g_{n} \text { in } \omega \\
\operatorname{div} u_{n}=0 \text { in } \omega,
\end{array}\right.
$$

where $e\left(u_{n}\right)$ is the symmetrized gradient of $u_{n}$, i.e.

$$
e\left(u_{n}\right)=\frac{1}{2}\left(D u_{n}+D u_{n}^{T}\right)
$$

and $C_{n} \in L^{\infty}\left(\Omega ; \mathcal{L}_{s}\left(\mathbb{R}_{s}^{2 \times 2} \cap \mathbb{R}_{0}^{2 \times 2}\right)\right)$ satisfies for some $\alpha>0$,

$$
C_{n}(x) \zeta: \zeta \geq \alpha|\zeta|^{2}, \quad \forall \zeta \in \mathbb{R}_{s}^{2 \times 2} \cap \mathbb{R}_{0}^{2 \times 2} \text {, a.e. } x \in \omega, \forall n \in \mathbb{N} \text {. }
$$

Then, defining $B_{n}$ by

$$
B_{n} \xi=\frac{1}{2} C_{n}\left(\xi+\xi^{T}\right), \quad \forall \xi \in \mathbb{R}_{0}^{2 \times 2} \text {, a.e. } x \in \omega, \forall n \in \mathbb{N},
$$

and assuming that $C_{n}$ is bounded in $L^{1}\left(\Omega ; \mathcal{L}_{s}\left(\mathbb{R}_{s}^{2 \times 2} \cap \mathbb{R}_{0}^{2 \times 2}\right)\right)$, we deduce that $B_{n}$ satisfies $(3.1)$, (3.2), and (3.3) by the Korn inequality.

Similarly as we did for the plate equation we introduce the following notation:

Definition 3.4. For an open set $\omega \subset \Omega$, we denote by $\mathcal{S}(\omega)$ the space of the functions $u$ in $H_{\text {div }}^{1}(\omega)$ such that there exists a sequence $u_{n} \in H_{\text {div }}^{1}(\omega)$ satisfying

$$
u_{n} \rightarrow u \text { in } L^{2}\left(\omega ; \mathbb{R}^{2}\right), \quad \limsup _{n \rightarrow \infty} \int_{\omega} B_{n} D u_{n}: D u_{n} d x<\infty
$$

and by $\mathcal{S}_{c}(\omega)$ the space of the functions $u$ in $\mathcal{S}(\omega)$ such that there exists a sequence $u_{n} \in H_{\text {div }}^{1}(\omega)$ satisfying (2.5), with $\operatorname{supp}\left(u_{n}\right)$ contained in a compact subset of $\omega$ independent of $n$.

We also denote by $D \mathcal{S}(\omega)$ and $D \mathcal{S}_{c}(\omega)$ the spaces

$$
D \mathcal{S}(\omega)=\{D u: u \in \mathcal{S}(\omega)\} \quad \text { and } \quad D \mathcal{S}_{c}(\omega)=\left\{D u: u \in \mathcal{S}_{c}(\omega)\right\}
$$

Then, we have the following result:

Theorem 3.5. There exists a subsequence of $n$, still denoted by $n$, and a family of symmetric bilinear applications $\mathfrak{b}_{\omega}: D \mathcal{S}(\omega) \times D \mathcal{S}(\omega) \rightarrow \mathcal{M}(\omega)$, for any open set $\omega \subset \Omega$, with the following properties:

- The operator $\mathfrak{b}_{\omega}$ is strongly local with respect to $\omega$ in the following sense: If $\omega^{1}$, $\omega^{2}$ are two open sets of $\omega$ and $u^{1}, v^{1} \in \mathcal{S}\left(\omega^{1}\right), u^{2}, v^{2} \in \mathcal{S}\left(\omega^{2}\right)$ are such that $D u^{1}=D u^{2}, D v^{1}=D v^{2}$ a.e. in $\omega^{1} \cap \omega^{2}$, then

$$
\mathfrak{b}_{\omega^{1}}\left(D u^{1}, D v^{1}\right)=\mathfrak{b}_{\omega^{2}}\left(D u^{2}, D v^{2}\right) \text { in } \omega^{1} \cap \omega^{2}
$$

- For any open set $\omega \subset \Omega$, and any $u_{n}, u \in H_{\mathrm{div}}^{1}(\omega)$ satisfying (3.10) and (3.4) for some sequence $g_{n}$ converging strongly to $g$ in $H^{-1}\left(\omega ; \mathbb{R}^{2}\right)$, the function $u$ satisfies

$$
\int_{\omega} d \mathfrak{b}_{\omega}(D u, D v)=\langle g, v\rangle, \quad \forall v \in \mathcal{S}_{c}(\omega)
$$


- Consider an open set $\omega \subset \Omega$ and $u_{n}, u \in H_{\text {div }}^{1}(\omega)$ satisfying (3.10) and

$$
\int_{\omega} B_{n} D u_{n}: D v_{n} d x \rightarrow 0
$$

for any $v_{n} \in H_{\mathrm{div}}^{1}(\omega)$, with support in a fixed compact of $\omega$, such that

$$
v_{n} \rightarrow 0 \quad \text { in } L^{2}\left(\omega ; \mathbb{R}^{2}\right), \quad \limsup _{n \rightarrow \infty} \int_{\omega} B_{n} D v_{n}: D v_{n} d x<\infty .
$$

Then, we have

$$
B_{n} D u_{n}: D v_{n} \stackrel{*}{\longrightarrow} \mathfrak{b}_{\omega}(D u, D v) \text { in } \mathcal{M}(\omega),
$$

for any $v_{n}, v \in H_{\operatorname{div}}^{1}(\omega)$ such that

$$
v_{n} \rightarrow v \quad \text { in } L^{2}\left(\omega ; \mathbb{R}^{2}\right), \quad \limsup _{n \rightarrow \infty} \int_{\omega} B_{n} D v_{n}: D v_{n} d x<\infty .
$$

- For any open set $\omega \subset \Omega$, the sequence of functionals $H_{n}^{\omega}$ defined by

$$
H_{n}^{\omega}(u)= \begin{cases}\int_{\omega} B_{n} D u: D u d x & \text { if } u \in H_{0, \mathrm{div}}^{1}(\omega) \\ \infty & \text { if } u \in L^{2}\left(\omega ; \mathbb{R}^{2}\right) \backslash H_{0, \mathrm{div}}^{1}(\omega) .\end{cases}
$$

Г-converges (up to a subsequence) to a functional $H^{\omega}$ which satisfies

$$
H^{\omega}(u)=\int_{\omega} d \mathfrak{b}_{\omega}(D u, D u), \quad \forall u \in \mathcal{S}_{c}(\omega)
$$

- The family $\mathfrak{b}_{\omega}$ satisfies the following ellipticity condition: For any open sets $O, U, \omega$ with $\bar{O} \subset U \subset \bar{U} \subset \omega \subset \Omega$, we have

$$
\beta(O, U) \int_{O}|D u|^{2} d x \leq \int_{\omega}|u|^{2} d x+\int_{\omega} d \mathfrak{b}_{\omega}(D u, D u), \quad \forall u \in \mathcal{S}(\omega) .
$$

- For any open set $\omega \subset \Omega$ and any $u \in W_{\operatorname{div}}^{1, \infty}(\omega)$, we have

$$
\int_{\omega} d \mathfrak{b}_{\omega}(D u, D u) \leq \nu(\omega)\|D u\|_{L^{\infty}\left(\omega ; \mathbb{R}_{0}^{2 \times 2}\right)}^{2} .
$$

- The family $\mathfrak{b}_{\omega}$ satisfies the following continuity property: For any open set $\omega \subset \Omega$ and any sequence $u^{k} \in \mathcal{S}(\omega)$ which converges to some $u \in \mathcal{S}(\omega)$ in $L^{2}\left(\omega ; \mathbb{R}^{2}\right)$, with $\mathfrak{b}_{\omega}\left(D u^{k}, D u^{k}\right)$ bounded in $\mathcal{M}(\omega)$, we have

$$
\mathfrak{b}_{\omega}\left(D u^{k}, D v\right) \stackrel{*}{\longrightarrow} \mathfrak{b}_{\omega}(D u, D v) \quad \text { in } \mathcal{M}(\omega), \quad \forall v \in \mathcal{S}(\omega) .
$$

A representation theorem of $\mathfrak{b}_{\omega}$ for smooth functions is given by the following result:

Theorem 3.6. For any open set $\omega \subset \Omega$, there exists a linear operator $\mathcal{B}_{\omega}: D \mathcal{S}(\omega) \rightarrow$ $L_{\nu}^{1}\left(\omega ; \mathbb{R}_{0}^{2 \times 2}\right)$, with the following properties: 
- The operator $\mathcal{B}_{\omega}$ is strongly local with respect to $\omega$ in the following sense: If $\omega^{1}, \omega^{2}$ are two open subsets of $\Omega$ and $u^{1} \in \mathcal{S}\left(\omega^{1}\right), u^{2} \in \mathcal{S}\left(\omega^{2}\right)$ are such that $D u^{1}=D u^{2}$ a.e. in $\omega^{1} \cap \omega^{2}$, then

$$
\mathcal{B}_{\omega^{1}} D u^{1}=\mathcal{B}_{\omega^{2}} D u^{2} \quad \nu \text {-a.e. in } \omega^{1} \cap \omega^{2} .
$$

- The operators $\mathfrak{b}_{\omega}$ and $\mathcal{B}_{\omega}$ are related by

$$
\mathfrak{b}_{\omega}(D u, D v)=\mathcal{B}_{\omega} D u: D v \nu, \quad \forall u, v \in \mathcal{S}(\omega), v \in C^{1}(\omega) .
$$

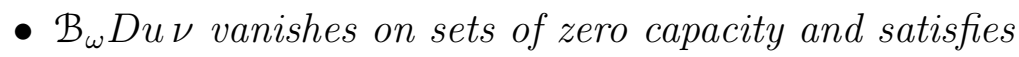

$$
\int_{\omega}\left|\mathcal{B}_{\omega} D u\right| \varphi d \nu \leq\left(\int_{\omega} \varphi d \mathfrak{b}_{\omega}(D u, D u)\right)^{\frac{1}{2}}\left(\int_{\omega} \varphi d \nu\right)^{\frac{1}{2}}, \forall \varphi \in C_{0}^{0}(\omega), \varphi \geq 0 .
$$

On the other hand, there exists a non-negative tensor $B \in L_{\nu}^{\infty}\left(\Omega ; \mathcal{L}_{s}\left(\mathbb{R}_{0}^{2 \times 2}\right)\right)$, with

$$
\|B\|_{L_{\nu}^{\infty}\left(\Omega ; \mathcal{L}_{s}\left(\mathbb{R}_{0}^{2 \times 2}\right)\right)} \leq 1 .
$$

such that for any $\omega \subset \Omega$ open and any $u \in C^{1}(\omega) \cap \mathcal{S}(\omega)$, we have

$$
\mathcal{B}_{\omega} D u=B D u \quad \nu \text {-a.e. in } \omega .
$$

Moreover, for any curve $\gamma$ of class $C^{2}$ contained in $\omega$, we have

$$
B(\mathrm{t} \otimes \mathrm{n}):(\mathrm{t} \otimes \mathrm{n})=0 \quad \nu \text {-a.e. in } \gamma,
$$

where $\mathrm{t}$ and $\mathrm{n}$ denote respectively the unitary tangent and normal vectors to $\gamma$.

Remark 3.7. Going back to the Stokes problem (3.4), the variational formulation (3.12) and formula (2.21) yield that the limit velocity $u$ is solution of

$$
\int_{\Omega} \mathcal{B}_{\omega} D u: D v \nu=\langle g, v\rangle, \quad \forall v \in C_{0}^{1}\left(\omega ; \mathbb{R}^{2}\right), \text { with } \operatorname{div} v=0 .
$$

Since $\mathcal{B}_{\omega} D u \nu$ is a zero trace matrix-valued measure, Corollary 2.10 of [8] implies the existence of a pressure $p \in H_{\mathrm{loc}}^{-1}(\omega) / \mathbb{R}$ such that $u$ and $p$ solve the Stokes equation in the distributions sense

$$
-\operatorname{div}\left(\mathcal{B}_{\omega} D u \nu\right)+\nabla p=g \text { in } \omega .
$$

By the same argument used in [8], the sequence $p_{n}$ is bounded in $H_{\text {loc }}^{-1}(\omega) / \mathbb{R}$, thus converges weakly to some $q$ in $H_{\text {loc }}^{-1}(\omega) / \mathbb{R}$. It follows that $p$ and $q$ agree in $H_{\text {loc }}^{-1}(\omega) / \mathbb{R}$. Therefore, we have just obtained the asymptotic of the Stokes problem (3.4), which was the motivation of the present section.

Remark 3.8. Similarly as we did in Proposition 2.8 for the plate equation, using property (3.27) the representation formula (3.26) can be extended to continuous functions which are piecewise $C^{1}$ in $\omega$. Indeed, if $u$ is a continuous function which is $C^{1}$ on both sides of a regular curve $\gamma$, then from the decomposition

$$
\begin{aligned}
D u= & ((D u \mathrm{t}) \cdot \mathrm{t})(\mathrm{t} \otimes \mathrm{t})+((D u \mathrm{n}) \cdot \mathrm{t})(\mathrm{t} \otimes \mathrm{n}) \\
& +((D u \mathrm{t}) \cdot \mathrm{n})(\mathrm{n} \otimes \mathrm{t})+((D u \mathrm{n}) \cdot \mathrm{n})(\mathrm{n} \otimes \mathrm{n}),
\end{aligned}
$$

taking into account that $D u \mathrm{t}$ is well defined and that $(D u \mathrm{n}) \cdot \mathrm{n}=-(D u \mathrm{t}) \cdot \mathrm{t}$ (as a consequence of $\operatorname{div} u=0)$, we deduce that $B D u \nu$ is well defined on $\gamma$ thanks to (3.27). 


\subsection{Proof of the results of Section 3}

Proof of Theorem 3.5. We apply Theorem 2.5 to the tensor $A_{n}$ given by (3.6).

For any open ball $B \subset \Omega$, we know (see, e.g., [19]) that there exists a linear continuous operator $R_{B}: H_{\text {div }}^{1}(B) \rightarrow H^{2}(B)$ such that

$$
J \nabla\left(R_{B} u\right)=u, \quad \forall u \in H_{\text {div }}^{1}(B) .
$$

Let us start by the following preliminary results:

1. By the definition of $A_{n}$, for any $u_{n}, v_{n} \in H_{\text {div }}^{1}(B)$, we have

$$
A_{n} D^{2} R_{B} u_{n}: D^{2} R_{B} v_{n}=B_{n} D u_{n}: D v_{n} \text { a.e. in } B \text {. }
$$

2. If $u \in \mathcal{S}(B)$, then for any $u_{n} \in H_{\text {div }}^{1}(B)$ satisfying (3.10), we have

$$
R_{B} u_{n} \rightarrow R_{B} u \text { in } L^{2}(B), \quad \limsup _{n \rightarrow \infty} \int_{B} A_{n} D^{2} R_{B} u_{n}: D^{2} R_{B} u_{n} d x<\infty
$$

which implies in particular that $R_{B} u$ belongs to $\mathcal{H}(B)$.

3. If the sequences $u_{n}, u \in H_{\text {div }}^{1}(B)$ satisfy (3.13) for any $v_{n} \in H_{\text {div }}^{1}(B)$ satisfying (3.14), then the sequences $R_{B} u_{n}, R_{B} u$ satisfy (2.11) for any sequence $z_{n} \in H^{2}(B)$ satisfying (2.12). Indeed, it is enough to apply (3.14) with $v_{n}=J D z_{n}$.

Let us now verify the properties of Theorem 3.5:

By the preliminary result 2 , for any open set $\omega \subset \Omega$, we may define the operator $\mathfrak{b}_{\omega}$ by

$$
\mathfrak{b}_{\omega}(D u, D v)=\mathfrak{a}_{B}\left(D^{2} R_{B} u, D^{2} R_{B} v\right) \text { in } B, \quad \forall B \text { open ball of } \omega .
$$

This definition is consistent thanks to the strong locality property satisfied by $\mathfrak{a}_{B}$. Moreover, definition (3.31) shows that the operator $\mathfrak{b}_{\omega}$ itself satisfies the strong local property (3.11).

Consider $u_{n}, u \in H_{\text {div }}^{1}(\omega)$ satisfying (3.13) and $v_{n}, v \in H_{\text {div }}^{1}(\omega)$ satisfying (3.14). Then, $u_{n}, u$ and $v_{n}, v$ satisfy these conditions for any open ball $B \subset \omega$. Hence, by the preliminary result 3 the functions $R_{B} u_{n}, R_{B} u$ satisfy (2.11), and $R_{B} v_{n}, R_{B} v$ (2.12). Therefore, by (2.13) we have

$$
A_{n} D^{2} R_{B} u_{n}: D^{2} R_{B} v_{n} \stackrel{*}{\longrightarrow} \mathfrak{a}_{\omega}\left(D^{2} R_{B} u, D^{2} R_{B} v\right) \text { in } \mathcal{M}(B), \quad \forall B \text { open ball of } \omega,
$$

which combined with definition (3.31) yields (3.15). Similarly, we can deduce (3.21) from (2.19).

Now, consider $u_{n}, u \in H_{\text {div }}^{1}(\omega)$ satisfying (3.10), and (3.4) for some sequence $g_{n}$ converging strongly to $g$ in $H^{-1}\left(\omega ; \mathbb{R}^{2}\right)$. Let $v \in \mathcal{S}_{c}(\omega)$, and let $v_{n} \in H_{\text {div }}^{1}(\omega)$ be a sequence satisfying (3.10), with $\operatorname{supp}\left(v_{n}\right)$ contained in a compact subset $K$ of $\omega$ independent of $n$. Let $\varphi \in C_{c}^{0}(\omega)$, with $\varphi \equiv 1$ in $K$. On the one hand, we have

$$
\int_{\omega} B_{n} D u_{n}: D v_{n} d x=\left\langle g_{n}, v_{n}\right\rangle \longrightarrow\langle g, v\rangle
$$

On the other hand, by (3.15) and the strong locality property of $\mathfrak{b}_{\omega}$, we have

$$
\int_{\omega} B_{n} D u_{n}: D v_{n} d x=\int_{\omega} B_{n} D u_{n}: D v_{n} \varphi d x \longrightarrow \int_{\omega} \varphi d \mathfrak{b}_{\omega}(D u, D v)=\int_{\omega} d \mathfrak{b}_{\omega}(D u, D v) .
$$

Equating the two limits we get (3.12). 
Next, consider a recovery sequence $\hat{u}_{n}$ (for $H_{n}^{\omega}$ ) of limit $u \in \mathcal{S}_{c}(\omega)$, and a sequence $u_{n}$ in $H_{\text {div }}^{1}(\omega)$ satisfying $(3.10)$, with $\operatorname{supp}\left(v_{n}\right)$ contained in a compact subset $K$ of $\omega$ independent of $n$. Let $\varphi \in C_{c}^{0}(\omega)$, with $\varphi \equiv 1$ in $K$. Again by (3.15) and the strong local property of $\mathfrak{b}_{\omega}$, we obtain that

$$
\begin{aligned}
H^{\omega}(u) & =\lim _{n \rightarrow \infty} \int_{\omega} B_{n} D \hat{u}_{n}: D \hat{u}_{n} d x=\lim _{n \rightarrow \infty} \int_{\omega} B_{n} D \hat{u}_{n}: D u_{n} d x \\
& =\lim _{n \rightarrow \infty} \int_{\omega} B_{n} D \hat{u}_{n}: D u_{n} \varphi d x=\int_{\omega} \varphi d \mathfrak{b}_{\omega}(D u, D u)=\int_{\omega} d \mathfrak{b}_{\omega}(D u, D u) .
\end{aligned}
$$

Finally, it remains to prove estimates (3.19) and (3.20). To this end, consider for $u \in \mathcal{S}(\omega)$ a recovery sequence of limit $u$ for the sequence $E_{n}$ defined by

$$
E_{n}(u)= \begin{cases}\int_{\omega} B_{n} D u: D u d x & \text { if } u \in H_{\mathrm{div}}^{1}(\omega) \\ \infty & \text { if } u \in L^{2}\left(\omega ; \mathbb{R}^{2}\right) \backslash H_{\mathrm{div}}^{1}(\omega) .\end{cases}
$$

Using (3.15) we then repeat the proofs of (2.17) and (2.18).

Proof of Theorem 3.6. Let $\omega$ be an open set of $\Omega$. Define the operator $\mathcal{B}_{\omega}$ by

$$
\mathcal{B}_{\omega} D u=J \mathcal{A}_{\omega}\left(D^{2} R_{B} u\right) \text { in } B, \quad \text { for } D u \in \mathcal{S}(\omega), \quad \forall B \text { open ball of } \omega,
$$

where $\mathcal{A}_{\omega}: D^{2} \mathcal{H}(\omega) \rightarrow L_{\mu}^{1}\left(\omega ; \mathbb{R}_{s}^{2 \times 2}\right)$ is the operator defined in Theorem 2.6, associated with the sequence $A_{n}$ given by (3.6). Since the measures $\mu$ and $\nu$ agree (as a consequence of (3.6)),

the formula (3.32) combined with (2.21) implies the relation (3.23) between the operators $\mathfrak{b}_{\omega}$ and $\mathcal{B}_{\omega}$. Similarly, using (3.32) the rest of the proof is a straightforward consequence of Theorem 2.6 and Proposition 2.8 for (3.27).

\section{A counter-example for the Stokes equation}

It was proved in [10] that a sequence of elastic energies the coefficients of which are not equibounded in $L^{1}$, may converge to a degenerate energy with a second gradient term. The counterexample of [10] is based on a reinforcement by very stiff strips parallel to the $x_{2}$-axis and periodically distributed in the domain $\Omega$. The sequence $u^{n}$ of displacements then converges weakly in $H_{0}^{1}(\Omega)$ to a function $u=\left(u_{1}, 0\right)$, where $u_{1}$ solves an equation of plate type with a fourth-order derivative.

The aim of this section is to construct an example in the case of the Stokes equation which leads us to a degeneracy. We cannot mimic the example of [10] since the equality $u_{2}=0$ combined with the incompressibility condition implies that the limit velocity is zero. The idea is to consider a reinforcement but concentrated on the line $x_{1}=0$. The difficulty of the analysis is to manage the incompressibility condition around the reinforcement strip. To this end we need a density result involving a wave equation (see Lemma 4.2 below).

We have the following result:

Theorem 4.1. Let $\Omega=(-1,1)^{2}$ and $f \in L^{2}(\Omega)^{2}$. Consider the sequence of reinforced Stokes problems

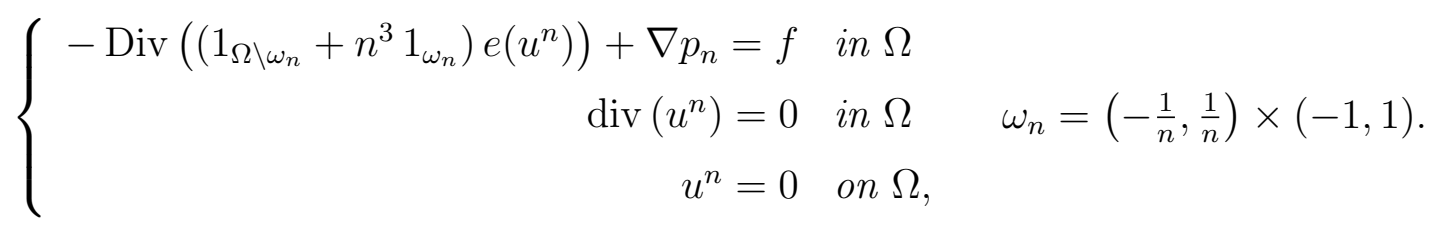


Then, the sequence of velocities $u^{n}$ converges weakly in $H_{0}^{1}(\Omega)^{2}$ to the function $u$ in the Hilbert space

$$
\begin{aligned}
& \quad V=\left\{v \in H_{0}^{1}(\Omega)^{2}: \operatorname{div}(v)=0, v_{2}(0, \cdot) \equiv 0, v_{1}(0, \cdot) \in H_{0}^{2}(-1,1)\right\}, \\
& \text { with }\|v\|_{V}=\|v\|_{H_{0}^{1}(\Omega)^{2}}+\left\|\partial_{22}^{2} v(0, \cdot)\right\|_{L^{2}(-1,1)}
\end{aligned}
$$

solution of the variational problem

$$
\int_{\Omega} e(u): e(v) d x+\frac{4}{3} \int_{-1}^{1} \partial_{22}^{2} u_{1}\left(0, x_{2}\right) \partial_{22}^{2} v_{1}\left(0, x_{2}\right) d x_{2}=\int_{\Omega} f \cdot v d x, \quad \forall v \in V .
$$

Proof. The fact that the viscosity in (4.1) is bounded from below by 1 combined with the Korn equality implies that the sequence $u^{n}$ is bounded in $H_{0}^{1}(\Omega)^{2}$, and has a bounded energy, i.e.

$$
\int_{\Omega \backslash \omega_{n}}\left|e\left(u^{n}\right)\right|^{2} d x+n^{3} \int_{\omega_{n}}\left|e\left(u^{n}\right)\right|^{2} d x \leq C .
$$

Therefore, up to a subsequence, still denoted by $n, u^{n}$ converges weakly converges to a divergence free function $u$ in $H_{0}^{1}(\Omega)^{2}$.

The proof is divided in two steps. In the first step we prove that $u$ belongs to the space $V$ (4.2) endowed with the norm $\|\cdot\|_{V}$. The second step is devoted to the derivation of the limit problem (4.3).

First step: Proof of $u \in V$.

Define the function $v^{n}$ in $\Omega$ by

$$
\left\{\begin{array}{l}
v_{1}^{n}\left(y_{1}, y_{2}\right)=u_{1}^{n}\left(\frac{y_{1}}{n}, y_{2}\right) \\
v_{2}^{n}\left(y_{1}, y_{2}\right)=n u_{2}^{n}\left(\frac{y_{1}}{n}, y_{2}\right),
\end{array} \quad \text { where } \quad\left(y_{1}, y_{2}\right)=\left(n x_{1}, x_{2}\right) \in \Omega\right.
$$

By this change of variables and the energy bound (4.4) we have

$$
n^{4} \int_{\Omega}\left(\partial_{1} v_{1}^{n}\right)^{2} d y+\frac{n^{2}}{4} \int_{\Omega}\left(\partial_{2} v_{1}^{n}+\partial_{1} v_{2}^{n}\right)^{2} d y+\int_{\Omega}\left(\partial_{2} v_{2}^{n}\right)^{2} d y=n^{3} \int_{\omega_{n}}\left|e\left(u^{n}\right)\right|^{2} d x \leq c,
$$

hence the sequence $e\left(v^{n}\right)$ is bounded in $L^{2}(\Omega)^{2 \times 2}$. This combined with the Dirichlet boundary condition $v^{n}\left(y_{1}, \pm 1\right)=0$, and the Korn inequality implies that $v^{n}$ is bounded in $H^{1}(\Omega)^{2}$. Therefore, up to a subsequence, still denoted by $n, v^{n}$ converges weakly to a function $v$ in $H^{1}(\Omega)$ satisfying $v\left(y_{1}, \pm 1\right)=0$. From (4.6) we easily deduce that

$$
\partial_{1} v_{1}=\partial_{2} v_{1}+\partial_{1} v_{2}=0 \quad \text { a.e. in } \Omega
$$

which combined with $v\left(y_{1}, \pm 1\right)=0$, implies that

$$
v_{1}(y)=a\left(y_{2}\right) \in H_{0}^{1}(-1,1)
$$

Moreover, by virtue of the compact embedding of $H^{1}(\Omega)$ into $L^{2}(\{0\} \times(-1,1))$ and by equality (4.8) we have

$\int_{-1}^{1}\left(v_{1}^{n}\left(0, y_{2}\right)-a\left(y_{2}\right)\right)^{2} d y_{2}=\int_{-1}^{1}\left(u_{1}^{n}\left(0, x_{2}\right)-a\left(x_{2}\right)\right)^{2} d x_{2} \underset{\varepsilon \rightarrow 0}{\longrightarrow} 0=\int_{-1}^{1}\left(u_{1}\left(0, x_{2}\right)-a\left(x_{2}\right)\right)^{2} d x_{2}$ 
hence

$$
u_{1}(0, \cdot)=a \in H_{0}^{1}(-1,1) .
$$

On the other hand, the second equality of (4.7) and (4.8) yield

$$
v_{2}(y)=-a^{\prime}\left(y_{2}\right) y_{1}+b\left(y_{2}\right) \in H^{1}(\Omega) .
$$

Then, taking into account that $v_{2} \in H^{1}(\Omega)$ and $v_{2}\left(y_{1}, \pm 1\right)=0$, we get that $a^{\prime} \in H_{0}^{1}(-1,1)$. Hence, by (4.9) it follows that

$$
u_{1}(0, \cdot)=a \in H_{0}^{2}(-1,1) .
$$

Finally, from the estimate

$$
\int_{-1}^{1}\left(u_{2}^{n}\left(0, x_{2}\right)\right)^{2} d x_{2}=\frac{1}{n^{2}} \int_{-1}^{1}\left(v_{2}^{n}\left(0, y_{2}\right)\right)^{2} d y_{2}=O\left(n^{-2}\right),
$$

we deduce that $u_{2}(0, \cdot) \equiv 0$ in $(-1,1)$. Therefore, $u$ belongs to the space $V$.

Second step: Derivation of the limit problem (4.3).

Let $\varphi$ be a function in $V$ such that for a given $\varepsilon>0$,

$$
e_{12}(\varphi)=0 \text { in }(-\varepsilon, \varepsilon) \times(-1,1), \quad \varphi_{1} \in C^{\infty}([-\varepsilon, \varepsilon] \times[-1,1]), \quad \varphi_{1}(0, \cdot) \in C_{c}^{\infty}(-1,1) .
$$

Then, we have for $n>\varepsilon^{-1}$,

$$
n^{3} \int_{\omega_{n}} e\left(u^{n}\right): e(\varphi) d x=n^{3} \int_{\omega_{n}} \partial_{1} u_{1}^{n} \partial_{1} \varphi_{1} d x+n^{3} \int_{\omega_{n}} \partial_{2} u_{2}^{n} \partial_{2} \varphi_{2} d x .
$$

Moreover, making a second-order expansion for $\left|x_{1}\right| \leq n^{-1}$, and using the equalities $\operatorname{div} \varphi=0$ and $\varphi_{2}(0, \cdot) \equiv 0$, we have

$$
\begin{aligned}
\partial_{1} \varphi_{1}(x) & =\partial_{1} \varphi_{1}\left(0, x_{2}\right)+x_{1} \partial_{11}^{2} \varphi_{1}\left(0, x_{2}\right)+O\left(n^{-2}\right)=-\partial_{2} \varphi_{2}\left(0, x_{2}\right)+x_{1} \partial_{11}^{2} \varphi_{1}\left(0, x_{2}\right)+O\left(n^{-2}\right) \\
& =x_{1} \partial_{11}^{2} \varphi_{1}\left(0, x_{2}\right)+O\left(n^{-2}\right) \\
\partial_{2} \varphi_{2}(x) & =-\partial_{1} \varphi_{1}(x)=-x_{1} \partial_{11}^{2} \varphi_{1}\left(0, x_{2}\right)+O\left(n^{-2}\right) .
\end{aligned}
$$

Putting these estimates in (4.13) and applying the Cauchy-Schwarz inequality together with estimate (4.6) we obtain

$$
\begin{aligned}
n^{3} \int_{\omega_{n}} e\left(u^{n}\right): e(\varphi) d x & =n^{3} \int_{\omega_{n}}\left(\partial_{1} u_{1}^{n}-\partial_{2} u_{2}^{n}\right) \partial_{11}^{2} \varphi_{1}\left(0, x_{2}\right) x_{1} d x+O\left(n^{-1}\right) \\
& =2 n^{3} \int_{\omega_{n}} \partial_{1} u_{1}^{n} \partial_{11}^{2} \varphi_{1}\left(0, x_{2}\right) x_{1} d x+o(1) .
\end{aligned}
$$

Then, making the change of variables (4.5) in (4.14) we have

$$
n^{3} \int_{\omega_{n}} e\left(u^{n}\right): e(\varphi) d x=2 n^{2} \int_{\Omega} \partial_{1} v_{1}^{n} \partial_{11}^{2} \varphi_{1}\left(0, y_{2}\right) y_{1} d y+o(1) .
$$

Moreover, using the free divergence of $u^{n}$ and equalities (4.10), (4.11) we have

$$
n^{2} \partial_{1} v_{1}^{n}=-\partial_{2} v_{2}^{n} \longrightarrow-\partial_{2} v_{2}=\partial_{22}^{2} u_{1}\left(0, y_{2}\right) y_{1}-b^{\prime}\left(y_{2}\right) \quad \text { weakly in } L^{2}(\Omega) .
$$


Hence, passing to the limit in the right-hand side of (4.15) together with convergence (4.16) it follows that

$$
\begin{gathered}
n^{3} \int_{\omega_{n}} e\left(u^{n}\right): e(\varphi) d x \underset{\varepsilon \rightarrow 0}{\longrightarrow} \quad 2 \int_{\Omega}\left(\partial_{22}^{2} u_{1}\left(0, y_{2}\right) y_{1}-b^{\prime}\left(y_{2}\right)\right) \partial_{11}^{2} \varphi_{1}\left(0, y_{2}\right) y_{1} d y \\
=\frac{4}{3} \int_{-1}^{1} \partial_{22}^{2} u_{1}\left(0, y_{2}\right) \partial_{11}^{2} \varphi_{1}\left(0, y_{2}\right) d y_{2},
\end{gathered}
$$

taking into account that $\int_{-1}^{1} y_{1} d y_{1}=0$ and $\int_{-1}^{1} y_{1}^{2} d y_{1}=\frac{2}{3}$. However, the first condition of (4.12) and the free divergence of $\varphi$ imply that

$$
\partial_{22}^{2} \varphi_{1}+\partial_{21}^{2} \varphi_{2}=\partial_{11}^{2} \varphi_{1}+\partial_{12}^{2} \varphi_{2}=0 \quad \text { in }(\varepsilon, \varepsilon) \times(-1,1)
$$

hence $\partial_{11}^{2} \varphi_{1}(0, \cdot) \equiv \partial_{22}^{2} \varphi_{1}(0, \cdot)$. Putting this equality in $(4.17)$ we get

$$
n^{3} \int_{\omega_{n}} e\left(u^{n}\right): e(\varphi) d x \underset{\varepsilon \rightarrow 0}{\longrightarrow} \frac{4}{3} \int_{-1}^{1} \partial_{22}^{2} u_{1}\left(0, y_{2}\right) \partial_{22}^{2} \varphi_{1}\left(0, y_{2}\right) d y_{2} .
$$

Now, putting $\varphi$ as test function in the variational form of the Stokes equation (4.1) we have

$$
\int_{\Omega \backslash \omega_{n}} e\left(u^{n}\right): e(\varphi) d x+n^{3} \int_{\omega_{n}} e\left(u^{n}\right): e(\varphi) d x=\int_{\Omega} f \cdot \varphi d x,
$$

where clearly $1_{\Omega \backslash \omega_{n}} e\left(u^{n}\right)$ converges weakly to $e(u)$ in $L^{2}(\Omega)^{2 \times 2}$. Therefore, passing to the limit in the previous equality together with (4.18) we deduce the variational formulation (4.3) for any $\varphi \in V$ satisfying the conditions (4.12). However, Lemma 4.2 below shows that the set of such functions $\varphi$ is dense in the space $V$ of (4.2), which concludes the proof of Theorem 4.1.

Lemma 4.2. Let $u=\left(u_{1}, u_{2}\right)$ be a function in $V$. Then, there exists a sequence $u^{\varepsilon} \in H_{0}^{1}(\Omega)$ with the following properties

$$
\begin{gathered}
u_{1}^{\varepsilon} \in C^{\infty}([-\varepsilon, \varepsilon] \times[-1,1]), \quad u_{1}^{\varepsilon}(0, \cdot) \in C_{c}^{\infty}(-1,1), \quad u_{2}^{\varepsilon}(0, \cdot) \equiv 0, \\
\operatorname{div}\left(u^{\varepsilon}\right)=0 \text { a.e. in } \Omega, \quad e_{12}\left(u^{\varepsilon}\right)=0 \text { a.e. in }(-\varepsilon, \varepsilon) \times(-1,1), \\
u^{\varepsilon} \longrightarrow u \text { strongly in } H_{0}^{1}(\Omega), \quad u_{1}^{\varepsilon}(0, \cdot) \longrightarrow u_{1}(0, \cdot) \text { strongly in } H_{0}^{2}(-1,1) .
\end{gathered}
$$

Proof. Let $\rho_{\delta} \in C^{\infty}(\mathbb{R})$ be a mollifier with compact support in $(-\delta / 2, \delta / 2)$, for $\delta \in(0,1)$. Since the functions

$$
u^{\delta}(x)=u\left(\frac{x_{1}}{1-\delta}, \frac{x_{2}}{1-\delta}\right) 1_{\left\{\max \left(\left|x_{1}\right|,\left|x_{2}\right|\right)<1-\delta\right\}}, \quad \tilde{u}^{\delta}(x)=\int_{\mathbb{R}} \rho_{\delta}\left(x_{2}-y_{2}\right) u^{\delta}\left(x_{1}, y_{2}\right) d y_{2}
$$

are divergence free in $\Omega, \tilde{u}_{2}^{\delta}(0, \cdot) \equiv 0$, and as $\delta$ tends to zero, $\tilde{u}^{\delta}$ converges strongly to $u$ in $H_{0}^{1}(\Omega)^{2}$ and $\tilde{u}^{\delta}(0, \cdot)$ converges strongly to $u(0, \cdot)$ in $H_{0}^{2}(-1,1)$, we can assume that $u$ has a compact support in $\Omega$ and $u_{1}(0, \cdot) \in C_{c}^{\infty}(-1,1)$. Then, defining

$$
\hat{u}^{\delta}\left(x_{1}, x_{2}\right)= \begin{cases}u\left(0, x_{2}\right) & \text { if }\left|x_{1}\right|<\delta \\ u\left(x_{1}-\delta, x_{2}\right) & \text { if } x_{1}>\delta \\ u\left(x_{1}+\delta, x_{2}\right) & \text { if } x_{1}<-\delta\end{cases}
$$

and taking into account as above that $\hat{u}^{\delta}$ is divergence free, and as $\delta$ tends to zero, $\hat{u}^{\delta}$ converges strongly to $u$ in $H_{0}^{1}(\Omega)^{2}$ with $\hat{u}^{\delta}(0, \cdot) \equiv u(0, \cdot)$, we can also assume that there exists $\delta>0$ such that $u$ does not depend on $x_{1}$ for $\left|x_{1}\right|<\delta$. 
From now on we assume that there exists $\delta>0$ such that $u(x)=0$ if $\max \left(\left|x_{1}\right|,\left|x_{2}\right|\right)>1-\delta$, $u$ does not depend on $x_{1}$ if $\left|x_{1}\right|<\delta$, and $u_{1}(0, \cdot) \in C_{c}^{\infty}(-1,1)$. In order to prove the lemma it is enough to show that for $0<\varepsilon<\delta$, there exists a function $u^{\varepsilon}=u+v^{\varepsilon}$ which converges strongly to $u$ in $H_{0}^{1}(\Omega)^{2}$ and satisfies

$\operatorname{div}\left(v^{\varepsilon}\right)=0$ in $\Omega, \quad v_{1}^{\varepsilon} \in C^{\infty}([-\varepsilon, \varepsilon] \times[-1,1]), \quad v^{\varepsilon}(0, \cdot) \equiv 0, \quad e_{12}\left(u^{\varepsilon}\right)=0$ in $(-\varepsilon, \varepsilon) \times(-1,1)$.

The function $v^{\varepsilon}$ must satisfy

$$
\partial_{1} v_{1}^{\varepsilon}+\partial_{2} v_{2}^{\varepsilon}=0, \quad \partial_{1} v_{2}^{\varepsilon}+\partial_{2} v_{1}^{\varepsilon}=-\partial_{2} u_{1}\left(0, x_{2}\right) \quad \text { in }(-\delta, \delta) \times(-1,1) .
$$

In particular $v_{2}^{\varepsilon}$ solves the wave equation

$$
\partial_{1}^{2} v_{\varepsilon}^{2}-\partial_{2}^{2} v_{2}^{\varepsilon}=0 \quad \text { in }(-\varepsilon, \varepsilon) \times(-1,1) .
$$

Therefore, $v^{\varepsilon}$ is of the form $v^{\varepsilon}(x)=a\left(x_{1}+x_{2}\right)+b\left(x_{1}-x_{2}\right)$. Using this expression in (4.19) and taking into account that $v^{\varepsilon}(0, \cdot) \equiv 0$, we get that $v_{\varepsilon}$ must be given by

$$
\left\{\begin{array}{l}
v_{1}^{\varepsilon}(x)=\frac{1}{2} u_{1}\left(0, x_{1}+x_{2}\right)+\frac{1}{2} u_{1}\left(0, x_{2}-x_{1}\right)-u_{1}\left(0, x_{2}\right) \\
v_{2}^{\varepsilon}(x)=-\frac{1}{2} u_{1}\left(0, x_{1}+x_{2}\right)+\frac{1}{2} u_{1}\left(0, x_{2}-x_{1}\right)
\end{array} \quad \text { in }[-\varepsilon, \varepsilon] \times[-1,1] .\right.
$$

Observe that $v^{\varepsilon}\left(x_{1}, x_{2}\right)=0$ on $\partial \Omega \cap\left\{\left|x_{1}\right| \leq \varepsilon\right\}$.

In order to define $v^{\varepsilon}$ in $\Omega^{\varepsilon}=\Omega \cap\left\{\left|x_{1}\right|>\varepsilon\right\}$, we consider the function $z^{\varepsilon} \in H^{1}\left(\Omega^{\varepsilon}\right)^{2}$ solution of the problem

$$
\left\{\begin{array}{l}
\Delta z^{\varepsilon}=0 \text { in } \Omega^{\varepsilon} \\
z^{\varepsilon}=0 \text { on } \partial \Omega^{\varepsilon} \cap\left\{\left|x_{1}\right|>\varepsilon\right\}, \quad z^{\varepsilon}\left(\varepsilon, x_{2}\right)=v^{\varepsilon}\left(\varepsilon, x_{2}\right), \quad z^{\varepsilon}\left(-\varepsilon, x_{2}\right)=v^{\varepsilon}\left(-\varepsilon, x_{2}\right) .
\end{array}\right.
$$

Taking into account the expression of $v^{\varepsilon}$, the sequence $z^{\varepsilon}$ satisfies

$$
\left\|z^{\varepsilon}\right\|_{H^{1}\left(\Omega^{\varepsilon}\right)} \underset{\varepsilon \rightarrow 0}{\longrightarrow} 0
$$

Moreover,

$$
\begin{aligned}
& \int_{\Omega^{\varepsilon} \cap\left\{x_{1}>\varepsilon\right\}} \operatorname{div}\left(z^{\varepsilon}\right) d x=-\int_{-1}^{1} v_{1}^{\varepsilon}\left(\varepsilon, x_{2}\right) d x_{2} \\
& =\frac{1}{2} \int_{-1}^{1} u_{1}\left(0, \varepsilon+x_{2}\right) d x_{2}+\frac{1}{2} \int_{-1}^{1} u_{1}\left(0, x_{2}-\varepsilon\right) d x_{2}-\int_{-1}^{1} u_{1}\left(0, x_{2}\right) d x_{2},
\end{aligned}
$$

but since $u\left(0, x_{2}\right)=0$ in $\left\{\left|x_{2}\right|>1-\varepsilon\right\}$ we have

$$
\int_{-1}^{1} u_{1}\left(0, \varepsilon+x_{2}\right) d x_{2}=\int_{-1}^{1} u_{1}\left(0, x_{2}-\varepsilon\right) d x_{2}=\int_{-1}^{1} u_{1}\left(0, x_{2}\right) d x_{2}=0 .
$$

Therefore, we obtain the equality

$$
\int_{\Omega^{\varepsilon} \cap\left\{x_{1}>\varepsilon\right\}} \operatorname{div}\left(z^{\varepsilon}\right) d x=0 .
$$

Similarly, we have

$$
\int_{\Omega^{\varepsilon} \cap\left\{x_{1}<-\varepsilon\right\}} \operatorname{div}\left(z^{\varepsilon}\right) d x=0 .
$$


This implies the existence of $w^{\varepsilon} \in H_{0}^{1}\left(\Omega^{\varepsilon}\right)$ satisfying

$$
\left\|w^{\varepsilon}\right\|_{H^{1}\left(\Omega^{\varepsilon}\right)^{2}} \leq C\left\|\operatorname{div}\left(z^{\varepsilon}\right)\right\|_{L^{2}\left(\Omega^{\varepsilon}\right)}, \quad \operatorname{div} w^{\varepsilon}=\operatorname{div}\left(u^{\varepsilon}\right) \text { in } \Omega^{\varepsilon} .
$$

Therefore, defining $v^{\varepsilon}$ by (4.20) in $\left\{\left|x_{1}\right| \leq \varepsilon\right\}$ and $v^{\varepsilon}=z^{\varepsilon}-w^{\varepsilon}$ in $\Omega^{\varepsilon}$, the function $u^{\varepsilon}=u+v^{\varepsilon}$ satisfies the conditions of Lemma 4.2 .

Acknowledgement: The authors wish to thank F. Murat for stimulating discussions about the counter-example of the last section. They also thank the unknown referee for his/her suggestions which have improved the paper.

The authors are grateful for support from the Spanish Ministerio de Economía y Competitividad through Project MTM2011-24457. This work was partly carried out while M. Briane was visiting the Department of Mathematics of the University of Sevilla in February 2011.

\section{References}

[1] M. Bellieud \& G. Bouchitté, "Homogenization of elliptic problems in a fiber reinforced structure. Nonlocal effects", Ann. Scuola Norm. Sup. Pisa Cl. Sci., 26 (4) (1998), 407-436.

[2] A. Beurling \& J. Deny, "Espaces de Dirichlet", Acta Matematica, 99 (1958), 203-224.

[3] J. Bourgain \& H. Brezis: "New estimates for elliptic equations and Hodge type systems", J. Eur. Math. Soc., 9 (2007), 277-315.

[4] A. BRAides: Г-convergence for Beginners, Oxford University Press, Oxford 2002, pp. 218.

[5] M. Briane, "Nonlocal effects in two-dimensional conductivity", Arch. Rat. Mech. Anal., 182 (2) (2006), 255-267.

[6] M. Briane \& J. Casado-Díaz, "Two-dimensional div-curl results. Application to the lack of nonlocal effects in homogenization", Com. Part. Diff. Equ., 32 (2007), 935-969.

[7] M. Briane \& J. Casado-Díaz, "Asymptotic behavior of equicoercive diffusion energies in dimension two", Calc. Var. PDE's, 29 (4) (2007), 455-479.

[8] M. Briane \& J. Casado-Díaz: "Estimate of the pressure when its gradient is the divergence of a measure. Applications.", to appear in ESAIM COCV, online: DOI:10.1051/cocv/2010037.

[9] M. Briane, J. Casado-Díaz \& F. Murat: "The div-curl lemma 'trente ans après': an extension and an application to the G-convergence of unbounded monotone operators", $J$. Math. Pures Appl., 91 (2009) 476?494.

[10] M. Briane \& M. Camar-Eddine: "Homogenization of two-dimensional elasticity problems with very stiff coefficients", J. Math. Pures Appl., 88 (2007), 483-505.

[11] M. Briane \& N. TChou, "Fibered microstructures for some nonlocal Dirichlet forms", Ann. Scuola Norm. Sup. Pisa Cl. Sci., 30 (4) (2001), 681-711.

[12] G. Dal Maso: An introduction to Г-convergence, Birkhaüser, Boston 1993, pp. 340. 
[13] M. Camar-Eddine \& P. Seppecher, "Closure of the set of diffusion functionals with respect to the Mosco-convergence", Math. Models Methods Appl. Sci., 12 (8) (2002), 11531176.

[14] M. Camar-Eddine \& P. Seppecher, "Determination of the closure of the set of elasticity functionals", Arch. Rat. Mech. Anal., 170 (3) (2003), 211-245.

[15] A. Damlamian \& M. Vogelius: "Homogenization limits of the equations of elasticity in thin domains", SIAM J. Math. Anal., 18 (2) (1987), 435-451.

[16] G. Duvaut \& A.-M. Metellus: "Homogénéisation d'une plaque mince en flexion de structure périodique et symétrique", C. R. Acad. Sci. Paris Sér. A-B, 283 (13) (1976), 947-950.

[17] V.N. Fenchenko \& E.Ya. Khruslov, "Asymptotic of solution of differential equations with strongly oscillating matrix of coefficients which does not satisfy the condition of uniform boundedness", Dokl. AN Ukr.SSR, 4 (1981).

[18] D. Gilbarg \& N. Trudinger: Elliptic Partial Differential Equations of Second Order, Springer, Berlin 2001, pp. 517.

[19] V. Girault \& P.-A. Raviart: Finite Element Approximation of the Navier-Stokes Equations, Lecture Notes in Mathematics, ed. by A. Dold \& B. Eckmann, 749, SpringerVerlag, Berlin Heidelberg New-York 1979.

[20] P.-L. Lions: "The concentration compactness principle in the calculus of variations. The limit case, part 1", Rev. Mat. Iber., 1 (1) (1985), 145-201.

[21] U. Mosco: "Composite media and asymptotic Dirichlet forms", J. of Functional Analysis, 123 (2) (1994), 368-421.

[22] F. Murat \& L. TARTAR: "H-convergence", Topics in the Mathematical Modelling of Composite Materials, L. Cherkaev and R.V. Kohn ed., Progress in Nonlinear Differential Equations and their Applications, Birkaüser, Boston 1998, 21-43.

[23] M.-J. Strauss: "Variations of Korn's and Sobolev's inequalities", Partial Differential Equations: Proc. Symp. Pure Math., 23, D. Spencer Ed., Amer. Math. Soc., Providence 1973, 207-214. 